\title{
Concentration and moderate deviations for Poisson polytopes and polyhedra
}

\author{
JULIAN GROTE* and CHRISTOPH THÄLE** \\ Ruhr University Bochum, Faculty of Mathematics, D-44780 Bochum, Germany.

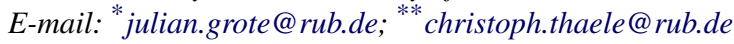

The convex hull generated by the restriction to the unit ball of a stationary Poisson point process in the $d$-dimensional Euclidean space is considered. By establishing sharp bounds on cumulants, exponential estimates for large deviation probabilities are derived and the relative error in the central limit theorem on a logarithmic scale is investigated for a large class of key geometric characteristics. This includes the number of lower-dimensional faces and the intrinsic volumes of the random polytopes. Furthermore, moderate deviation principles for the spatial empirical measures induced by these functionals are also established using the method of cumulants. The results are applied to a class of zero cells associated with Poisson hyperplane mosaics. As a special case, this comprises the typical Poisson-Voronoi cell conditioned on having large inradius.

Keywords: concentration inequalities; convex hulls; cumulants; deviation probabilities; moderate deviation principles; Poisson hyperplanes; Poisson-Voronoi mosaics; random polytopes; zero cells

\section{Introduction}

Random polytopes are among the most classical and popular models considered in geometric probability, and their study has become a rapidly developing branch of mathematics at the borderline between geometry and probability. One reason for the increasing interest are the numerous connections and applications of random polytopes in algorithmic geometry, convex geometric analysis, optimization, random matrix theory, set estimation or multivariate statistics; we direct the reader to the surveys of Bárány [4], Hug [26], Reitzner [35] and to Chapter 11 of the monograph of Brazitikos, Giannopoulos, Valettas and Vritsiou [10] for further information and references.

A common method to construct a random polytope is to take the convex hull of a finite family of random points that are uniformly distributed in the interior of a prescribed convex body $K \subset \mathbb{R}^{d}$ with $d \geq 2$. In their seminal paper, Rényi and Sulanke [36] considered the asymptotic behaviour of the mean vertex number and the mean volume (area) of such random polytopes if $d=2$, as the number of points tends to infinity. Since then, first-order asymptotic properties of geometric characteristics of random polytopes have been investigated for general space dimensions by Bárány [2,3], Böröczky, Hoffmann and Hug [9], Reitzner [32,33] or Schütt [42], to name only a few. More recently, the focus has turned towards asymptotic second-order characteristics like the variance of the number of vertices or the variance of the volume. The classical Efron-Stein inequality has been used by Reitzner [31] to obtain upper bounds for these variances 
as well as laws of large numbers. Matching lower bounds together with related central limit theorems have been shown on different levels of generality by Bárány and Reitzner [5,6], Cabo and Groeneboom [12], Groeneboom [21], Hueter [25], Pardon [30], Reitzner [34], Schreiber [39] and $\mathrm{Vu}$ [43]. This line of research has been continued in a series of remarkable papers by Calka, Schreiber and Yukich [16], Calka and Yukich [17] and Schreiber and Yukich [41].

In contrast to the typical or "normal" behaviour of random polytopes, much less is known about their atypical or exceptional behaviour, or at scales in between. For random polytopes in the unit ball, Calka and Schreiber [15] have obtained information on large deviations for the vertex number and Schreiber [40] has computed certain moderate deviation probabilities for the mean width. Moreover, the paper of $\mathrm{Vu}$ [44] deals in a general context with concentration inequalities for the volume and the vertex number. Besides such large deviation or concentration inequalities, it is from a probabilistic point of view also natural to ask for the behaviour of geometric characteristics associated with random polytopes on intermediate scales "between" that of the above-mentioned law of large numbers and that of a central limit theorem. The present paper is an attempt to fill this gap and to prove a set of concentration inequalities in the case where the underlying convex body $K$ is the $d$-dimensional unit ball $\mathbb{B}^{d}$ and where the family of random points is induced by a Poisson point process (Poisson polytopes). We refer to the papers of Affentranger [1], Buchta and Müller [11], Calka and Schreiber [15], Hsing [24], Küfer [28], Müller [29] and Schreiber [39,40] for distinguished results about random polytopes in $\mathbb{B}^{d}$.

Consider a stationary Poisson point process in $\mathbb{R}^{d}$ with intensity $\lambda>0$, let $\eta_{\lambda}$ be its restriction to $\mathbb{B}^{d}$ and let $\Pi_{\lambda}$ be the convex hull of the points of $\eta_{\lambda}$. For simplicity and to facilitate access to our results, we restrict for the rest of this introduction to the vertex number $f_{0}\left(\Pi_{\lambda}\right)$ of the random polytopes $\Pi_{\lambda}$ and refer to Section 3 for theorems dealing with other geometric characteristics of $\Pi_{\lambda}$ as well. Our first theorem is a concentration inequality for the vertex number of the random polytopes $\Pi_{\lambda}$. In order to present it and also for later use, let us define the constant

$$
\beta(d):=4+\frac{2}{d+1} .
$$

Theorem 1.1. Let $y \geq 0$. Then, for $\lambda \geq c_{1}$,

$$
\mathbb{P}\left(\left|f_{0}\left(\Pi_{\lambda}\right)-\mathbb{E} f_{0}\left(\Pi_{\lambda}\right)\right| \geq y \sqrt{\operatorname{var} f_{0}\left(\Pi_{\lambda}\right)}\right) \leq 2 \exp \left(-\frac{1}{4} \min \left\{\frac{y^{2}}{2^{\beta(d)}}, c_{2} \lambda^{\frac{d-1}{2 \beta(d)(d+1)}} y^{\frac{1}{\beta(d)}}\right\}\right)
$$

with constants $c_{1}, c_{2} \in(0, \infty)$ only depending on $d$.

Theorem 1.1 should be compared with the only known result of this kind from the literature. Provided that $\lambda$ is sufficiently large, Theorem 2.11 in [44] says in our situation that

$$
\mathbb{P}\left(\left|f_{0}\left(\Pi_{\lambda}\right)-\mathbb{E} f_{0}\left(\Pi_{\lambda}\right)\right| \geq y \sqrt{\operatorname{var} f_{0}\left(\Pi_{\lambda}\right)}\right) \leq 2 \exp \left(-b_{1} y^{2}\right)+p_{\mathrm{NT}},
$$

for all $0<y<b_{2} \lambda^{-\frac{(d+3)^{2}}{(d+1)(3 d+5)}}$ with constants $b_{1}, b_{2} \in(0, \infty)$ only depending on $d$, see also [35] for a related version. Here, $p_{\mathrm{NT}}$ is the probability of what is called a "non-typical event" in [44] and satisfies the estimate $p_{\mathrm{NT}} \leq \exp \left(-b_{3} \lambda^{\frac{d-1}{3 d+5}}\right)$, independently of $y$, with another constant 
$b_{3} \in(0, \infty)$ depending only on $d$. Our theorem basically recovers the exponential term in Vu's inequality. However, while Vu's inequality involves the boundary term $p_{\mathrm{NT}}$, which does not depend on $y$, such a term is not present in Theorem 1.1. Furthermore, our inequality yields an exponential estimate for all $y \geq 0$ and not only for values of $y$ close to zero. We also emphasize that the inequality in Theorem 1.1 remains valid for a wide class of geometric functionals, while in [44] besides of $f_{0}\left(\Pi_{\lambda}\right)$ only the volume of $\Pi_{\lambda}$ is treated. On the other hand, Theorem 1.1 deals with the case of a random polytope in the unit ball, whereas in [44] arbitrary underlying convex bodies are permitted.

Our next result is an estimate for certain deviation probabilities on a logarithmic scale that characterize the relative error in the central limit theorem for the normalized vertex number. To state it, denote by $\Phi(\cdot)$ the distribution function of a standard Gaussian random variable.

Theorem 1.2. For $0 \leq y \leq c_{3} \lambda^{\frac{d-1}{2(d+1)(2 \beta(d)-1)}}$ and $\lambda \geq c_{4}$ one has that

$$
\begin{gathered}
\left|\log \frac{\mathbb{P}\left(f_{0}\left(\Pi_{\lambda}\right)-\mathbb{E} f_{0}\left(\Pi_{\lambda}\right) \geq y \sqrt{\operatorname{var} f_{0}\left(\Pi_{\lambda}\right)}\right)}{1-\Phi(y)}\right| \leq c_{5}\left(1+y^{3}\right) \lambda^{-\frac{d-1}{2(d+1)(2 \beta(d)-1)}} \quad \text { and } \\
\left|\log \frac{\mathbb{P}\left(f_{0}\left(\Pi_{\lambda}\right)-\mathbb{E} f_{0}\left(\Pi_{\lambda}\right) \leq-y \sqrt{\operatorname{var} f_{0}\left(\Pi_{\lambda}\right)}\right)}{\Phi(-y)}\right| \leq c_{5}\left(1+y^{3}\right) \lambda^{-\frac{d-1}{2(d+1)(2 \beta(d)-1)}}
\end{gathered}
$$

with constants $c_{3}, c_{4}, c_{5} \in(0, \infty)$ only depending on $d$.

Our next theorem makes a statement about moderate deviations of the rescaled vertex number of $\Pi_{\lambda}$, which can be regarded as a kind of refinement of a central limit theorem. We will see in Theorem 3.5 below that the set $B$ appearing in Theorem 1.3 can be replaced in a way by an arbitrary measurable subset $B \subset \mathbb{R}$ and that the rescaled vertex number of the random polytope $\Pi_{\lambda}$ satisfies a so-called moderate deviation principle.

Theorem 1.3. Let $\left(a_{\lambda}\right)_{\lambda>0}$ be such that

$$
\lim _{\lambda \rightarrow \infty} a_{\lambda}=\infty \quad \text { and } \quad \lim _{\lambda \rightarrow \infty} a_{\lambda} \lambda^{-\frac{d-1}{2(d+1)(2 \beta(d)-1)}}=0 .
$$

Then, for all $y \in \mathbb{R}$, one has that

$$
\lim _{\lambda \rightarrow \infty} \frac{1}{a_{\lambda}^{2}} \log \mathbb{P}\left(\frac{1}{a_{\lambda}} \frac{f_{0}\left(\Pi_{\lambda}\right)-\mathbb{E} f_{0}\left(\Pi_{\lambda}\right)}{\sqrt{\operatorname{var} f_{0}\left(\Pi_{\lambda}\right)}} \in B\right)=-\frac{y^{2}}{2} \quad \text { with } B=[y, \infty) .
$$

As anticipated above, we will see in Section 3 that Theorems 1.1, 1.2 and 1.3 continue to hold for a large class of key geometric functionals of the random polytopes $\Pi_{\lambda}$ (possibly under different rescalings and with different exponents). In particular, this includes

- the number of $j$-dimensional faces of $\Pi_{\lambda}$ for all $j \in\{0,1, \ldots, d-1\}$,

- the missed volume of $\Pi_{\lambda}$ in $\mathbb{B}^{d}$,

- the missed volume of the Voronoi-flower of $\Pi_{\lambda}$ in $\mathbb{B}^{d}$,

- the mean width of $\Pi_{\lambda}$ and, more generally, 
- the $j$ th intrinsic volume of $\Pi_{\lambda}$ for all $j \in\{1, \ldots, d-1\}$.

In addition, we will work on the level of empirical measures and thus take care also of the spatial profile of the involved functionals. This in turn puts us in a position to present our announced moderate deviation principle also on the level of measures. Let us emphasize at this point that Theorem 1.2 and its generalization in Theorem 3.2 as well as the moderate deviation principles in Theorems 3.5 and 3.6 seem to be the first results in this direction in the context of random polytopes and that we were not able to locate counterparts in the existing literature.

Let us briefly comment on the technique we use to derive Theorems 1.1-1.3 and their generalizations stated in Section 3. It is based on precise estimates of the cumulants of the involved random variables (see Proposition 5.1 below). The methodology to deduce fine probabilistic estimates from bounds on cumulants goes back to the large deviation theory summarized in the monograph of Saulis and Statulevičius [37]. In the context of geometric probability, this has been used by Eichelsbacher, Raič and Schreiber [20] to deduce results similar to those presented above for a class of so-called stabilizing functionals. However, the random polytope functionals we consider behave quite differently and are not within the reach of the results in [20]. Instead, we use the principal idea from $[16,41]$ that connects $\Pi_{\lambda}$ with a parabolic growth process in the upper half-space. The key advantage of this connection lies in the fact that in the rescaled parabolic picture spatial correlations are much easier to localize and to quantify. Even on the level of the central limit theorem all "traditional" proofs, not using the scaling transformation, rely on a conditioning argument, see [34], page 499, or [6], Section 7. However, it is not clear (to us) how such an argument can be used for our purposes. Against this background, the scaling transformation is an indispensable tool for us. We then adapt the methods from [7] and [20] and develop them further to make the cumulant approach available in the context of the random polytopes $\Pi_{\lambda}$ (see Remarks 5.4 and 5.6 below for a more detailed discussion). Our probabilistic estimates then follow from the main "lemmas" in [37] and the moderate deviations from the work of Döring and Eichelsbacher [19]. The main technical difficulty in carrying out this approach is that only the points of the Poisson point process $\eta_{\lambda}$ close to the boundary of $\mathbb{B}^{d}$ contribute to the geometry of $\Pi_{\lambda}$, an effect that cannot be handled within the framework of [20], but which is typical for random polytopes.

Instead of taking the convex hull of random points, it is also natural to consider random sets that arise as intersections of random half-spaces, see the surveys of Hug [26] and Reitzner [35]. To understand the geometric and the combinatorial structure of such random polyhedra is of importance, for example, in linear optimization. In particular, the performance of the well-known simplex algorithm depends on the number of edges of the polyhedron that is defined as the intersection of the set of half-space determined by a system of linear inequalities. One way to obtain a deeper insight into the generic combinatorial complexity that arises in such situations is to consider random polyhedral sets as argued in Borgwardt's monograph [8]. By a duality argument borrowed from the works of Calka and Schreiber [13,14] we transfer our results for random polytopes to combinatorial parameters of a certain class of random polyhedra that are associated with Poisson hyperplanes (Poisson polyhedra). In particular, this includes the prominent typical cell of a stationary Poisson-Voronoi tessellation of $\mathbb{R}^{d}$ conditioned on having a large inradius. In this context, we also contribute to the results around D.G. Kendall's conjecture asking for the asymptotic geometry of "large" tessellation cells and for which we refer in particular to the paper of Hug and Schneider [27] as well as to the references cited therein. 
The remaining parts of the paper are organized as follows. In Section 2, we introduce the formal framework and recall the necessary results from [16] in order to keep the presentation sufficiently self-contained. Our main theorems for Poisson polytopes are presented in full generality in Section 3, while the final Section 5 contains their proofs. In Section 4, we apply our main results to a parametric class of random polyhedra that arise from Poisson hyperplanes.

\section{Framework and background}

\subsection{Basic notions and notation}

In this paper, we write $V_{d}(\cdot)$ for the $d$-dimensional volume (Lebesgue measure) of the argument set. We denote the Euclidean scalar product by $(\cdot, \cdot)$, the norm induced by it by $\|\cdot\|$ and put $\mathbb{B}^{d}:=\left\{x \in \mathbb{R}^{d}:\|x\| \leq 1\right\}$ and $\mathbb{S}^{d-1}:=\left\{x \in \mathbb{R}^{d}:\|x\|=1\right\}$. We further indicate by $\mathbb{B}^{d}(x, r)$ the ball centered at $x \in \mathbb{R}^{d}$ with radius $r>0$ and define the constant $\kappa_{k}:=V_{k}\left(\mathbb{B}^{k}\right), k \in\{0,1,2, \ldots\}$. We denote by $\mathcal{H}_{\mathbb{S}^{d-1}}^{d-1}$ the $(d-1)$-dimensional Hausdorff measure on $\mathbb{S}^{d-1}$.

Let $\Sigma$ be a Polish space. By $\mathcal{B}(\Sigma)$ we denote the space of bounded measurable functions $f: \Sigma \rightarrow \mathbb{R}$ and we write $\mathcal{M}(\Sigma)$ for the space of finite signed measures on $\Sigma$. For $f \in \mathcal{B}(\Sigma)$ and $v \in \mathcal{M}(\Sigma)$ we introduce the abbreviation $\langle f, v\rangle:=\int \mathrm{d} v f$ for the integral of $f$ with respect to $v$. We will further use the symbol $\mathcal{C}(\Sigma)$ for the space of continuous functions on $\Sigma$.

By a convex body we understand a compact convex subset $K \subset \mathbb{R}^{d}$ with non-empty interior. For a convex body $K$ we denote by $V_{j}(K)$ the $j$ th intrinsic volume of $K, j \in\{0,1, \ldots, d-1\}$. In particular, $2 V_{d-1}(K)$ is the surface area and $V_{1}(K)$ is a constant multiple of the mean width of $K$, while $V_{0}(K)=1$. We further write $\operatorname{ext}(K)$ for the set of extreme points of $K$ and by the extreme points of a finite point set we understand the extreme points of its convex hull. For a polytope $P \subset \mathbb{R}^{d}$ and $j \in\{0,1, \ldots, d-1\}$, we write $f_{j}(P)$ for the number of $j$-dimensional faces of $P$.

One says that a family $\left(\nu_{\lambda}\right)_{\lambda>0}$ of probability measures on a Polish space $\Sigma$ fulfils a large deviation principle (LDP) with speed $s_{\lambda}$ and (good) rate function $I: \Sigma \rightarrow[0, \infty]$, as $\lambda \rightarrow \infty$, if $\lim _{\lambda \rightarrow \infty} s_{\lambda}=\infty, I$ is lower semi-continuous with compact level sets, and if

$$
-\inf _{y \in \operatorname{int}(B)} I(y) \leq \liminf _{\lambda \rightarrow \infty} \frac{1}{s_{\lambda}} \log v_{\lambda}(B) \leq \limsup _{\lambda \rightarrow \infty} \frac{1}{s_{\lambda}} \log v_{\lambda}(B) \leq-\inf _{y \in \operatorname{cl}(B)} I(y)
$$

for every measurable subset $B$ of $\Sigma$ with interior $\operatorname{int}(B)$ and closure $\operatorname{cl}(B)$, cf. [18]. A family $\left(X_{\lambda}\right)_{\lambda>0}$ of $\Sigma$-valued (and usually rescaled) random variables is said to satisfy a LDP with speed $s_{\lambda}$ and rate function $I$ if the family of their distributions does. One usually speaks about a moderate deviation principle (MDP) instead of a LDP if the rescaling of the involved random variables is between that of a law of large numbers and that of a central limit theorem.

\subsection{Key geometric functionals and their rescaled versions}

From now on, let $\eta_{\lambda}$ be the restriction to $\mathbb{B}^{d}$ of a stationary Poisson point process with intensity $\lambda>0$ and denote by $\Pi_{\lambda}$ the random convex hull generated by the points of $\eta_{\lambda}$. We recall from 


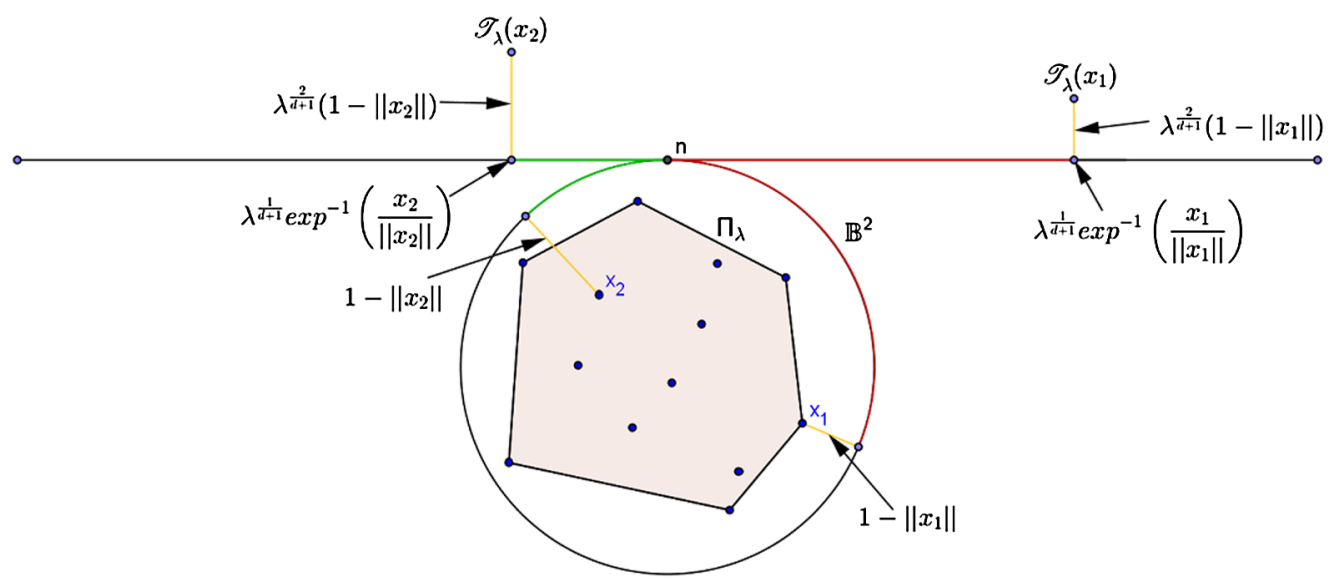

Figure 1. Illustration of the scaling transformation $\mathscr{T}_{\lambda}$.

[16] that each of the geometric characteristics $V_{j}\left(\mathbb{B}^{d}\right)-V_{j}\left(\Pi_{\lambda}\right)$ with $j \in\{1, \ldots, d\}$ and $f_{j}\left(\Pi_{\lambda}\right)$ with $j \in\{0, \ldots, d-1\}$ is representable as

$$
\sum_{x \in \eta_{\lambda}} \xi\left(x, \eta_{\lambda}\right)
$$

with certain explicitly known functionals $\xi$ (also called score functions) that we abbreviate by $\xi_{V_{j}}$ and $\xi_{f_{j}}$, respectively. Moreover, we recall that the Voronoi-flower $\operatorname{VF}\left(\eta_{\lambda}\right)$ of $\eta_{\lambda}\left(\right.$ or $\left.\Pi_{\lambda}\right)$ is given by $\operatorname{VF}\left(\eta_{\lambda}\right):=\bigcup_{x \in \eta_{\lambda}} \mathbb{B}^{d}\left(\frac{x}{2}, \frac{\|x\|}{2}\right)$. The Voronoi-flower of a random polytope is of interest because of the following observation. Writing $h_{K}(u):=\max \{(u, v): v \in K\}$ for the support function of a convex body $K$ in direction $u \in \mathbb{S}^{d-1}$, one has that the defect support function $1-h_{\Pi_{\lambda}}(u)$ of $\Pi_{\lambda}$ is precisely the distance between $\mathbb{S}^{d-1}$ and $\operatorname{VF}\left(\eta_{\lambda}\right)$ in direction $u$. It has been demonstrated in [16] that also the random variable $V_{d}\left(\mathbb{B}^{d}\right)-V_{d}\left(\operatorname{VF}\left(\eta_{\lambda}\right)\right)$ has a representation as in (3) with a suitable function $\xi$ there that we denote by $\xi_{\mathrm{VF}}$. The Voronoi-flower of $\Pi_{\lambda}$ is also a crucial object in Section 4. Moreover, we define $\Xi:=\left\{\xi_{\mathrm{VF}}, \xi_{V_{1}}, \ldots, \xi_{V_{d}}, \xi_{f_{0}}, \ldots, \xi_{f_{d-1}}\right\}$ to be the set of all key geometric functionals associated with $\Pi_{\lambda}$.

Let $n:=(0,0, \ldots, 1)$ be the north pole of $\mathbb{S}^{d-1}$ and identify the tangent space Tan $\left(\mathbb{S}^{d-1}, n\right)$ of $\mathbb{S}^{d-1}$ at $n$ with the $(d-1)$-dimensional Euclidean space $\mathbb{R}^{d-1}$. The exponential map exp : $\operatorname{Tan}\left(\mathbb{S}^{d-1}, n\right) \rightarrow \mathbb{S}^{d-1}$ transforms a vector $u \in \operatorname{Tan}\left(\mathbb{S}^{d-1}, n\right)$ into a point $\exp (u) \in \mathbb{S}^{d-1}$ such that $\exp (u)$ lies at the end of a geodesic ray of length $\|u\|$ and direction $u$ emanating from $n$, see Figure 1. In particular, $\exp (n)=0$. (The exponential map should not be confused with the exponential function which is denoted by the same symbol, but the meaning will always be clear from the context.) Although the exponential map is well defined on the whole tangent space, its injectivity region is $\mathbb{B}^{d-1}(0, \pi):=\left\{u \in \operatorname{Tan}\left(\mathbb{S}^{d-1}, n\right):\|u\|<\pi\right\}$, the centred (open) ball in $\mathbb{R}^{d-1}$ with radius $\pi$. Let us further denote by $\exp ^{-1}$ the inverse of the exponential map, which is well defined on $\mathbb{S}^{d-1} \backslash\{-n\}$. 
Following [16], we define the scaling transformation $\mathscr{T}_{\lambda}$ mapping $\mathbb{B}^{d}$ to $\mathbb{R}^{d-1} \times \mathbb{R}_{+}$by

$$
\mathscr{T}_{\lambda}(x):=\left(\lambda^{\frac{1}{d+1}} \exp ^{-1}\left(\frac{x}{\|x\|}\right), \lambda^{\frac{2}{d+1}}(1-\|x\|)\right), \quad x \in \mathbb{B}^{d} \backslash\{0\} .
$$

In particular, we notice that, by the well-known mapping properties of Poisson point processes, $\mathscr{T}_{\lambda}$ maps the Poisson point process $\eta_{\lambda}$ to another Poisson point process $\eta_{\lambda}^{\mathscr{T}}$ in the region

$$
\mathcal{R}_{\lambda}:=\lambda^{1 /(d+1)} \mathbb{B}^{d-1}(0, \pi) \times\left[0, \lambda^{2 /(d+1)}\right) \subset \mathbb{R}^{d-1} \times \mathbb{R}_{+}
$$

(note that with probability one, neither $-n$ nor 0 is a point of $\eta_{\lambda}$, meaning that the definition of exp and $\exp ^{-1}$ at these points is irrelevant). In what follows, we parametrize the points of $\mathbb{R}^{d-1} \times \mathbb{R}_{+}$as pairs $(v, h)$ with $v \in \mathbb{R}^{d-1}$ and $h \in \mathbb{R}_{+}$. Using this parametrization, it is known from equation (2.14) in [16] that the intensity measure of $\eta_{\lambda}^{\mathscr{T}}$ has density

$$
(v, h) \mapsto \frac{\sin ^{d-2}\left(\lambda^{-1 /(d+1)}\|v\|\right)}{\left\|\lambda^{-1 /(d+1)} v\right\|^{d-2}}\left(1-\lambda^{-2 /(d+1)} h\right)^{d-1}
$$

with respect to the Lebesgue measure on $\mathcal{R}_{\lambda}$. In particular, this implies that the limit process of $\eta_{\lambda}^{\mathscr{T}}$, as $\lambda \rightarrow \infty$, is a Poisson point process $\eta$ on the whole half-space $\mathbb{R}^{d-1} \times \mathbb{R}_{+}$whose intensity measure coincides with the Lebesgue measure on that space.

Using the scaling transformation $\mathscr{T}_{\lambda}$ we define the collection of rescaled key geometric functionals. Let $\xi \in \Xi$ and put

$$
\xi^{(\lambda)}(x, \mathcal{X}):=\xi\left(\mathscr{T}_{\lambda}^{-1}(x), \mathscr{T}_{\lambda}^{-1}(\mathcal{X})\right)
$$

for a locally finite point set $\mathcal{X}$ in the region $\mathcal{R}_{\lambda}$ and $x \in \mathcal{X}$. We define $\Xi^{(\lambda)}:=\left\{\xi^{(\lambda)}: \xi \in \Xi\right\}$ as the set of rescaled functionals.

One of the crucial features of the above scaling transformation is that the rescaled functionals $\xi^{(\lambda)}$ exhibit a weak spatial dependence property in the following sense. A random variable $R:=$ $R(\xi, x, \lambda)$ is called a radius of localization for $\xi^{(\lambda)}$ if, with probability one,

$$
\xi^{(\lambda)}\left(x, \eta_{\lambda}^{\mathscr{T}}\right)=\xi^{(\lambda)}\left(x, \eta_{\lambda}^{\mathscr{T}} \cap \operatorname{Cyl}(x, r)\right)
$$

for all $r \geq R$. Here, for a point $x=(v, h), \operatorname{Cyl}(x, r)$ stands for the cylinder $\mathbb{B}^{d-1}(v, r) \times \mathbb{R}_{+}$. Thus, the radius of localization does not depend on the height coordinate $h$ of the underlying point $x$. It has been shown in [16] that the infimum over all such $R$, which we also denote by $R$ to simplify the notation, has super-exponentially decaying tails uniformly in $x$ and $\lambda$. More formally, one can find constants $c_{1}, c_{2} \in(0, \infty)$ only depending on $\xi$ such that

$$
\mathbb{P}(R \geq u) \leq c_{1} \exp \left(-c_{1}^{-1} u^{d+1}\right), \quad u>0,
$$

for all $\lambda \geq c_{2}$, uniformly in $x$ and $\lambda$. 


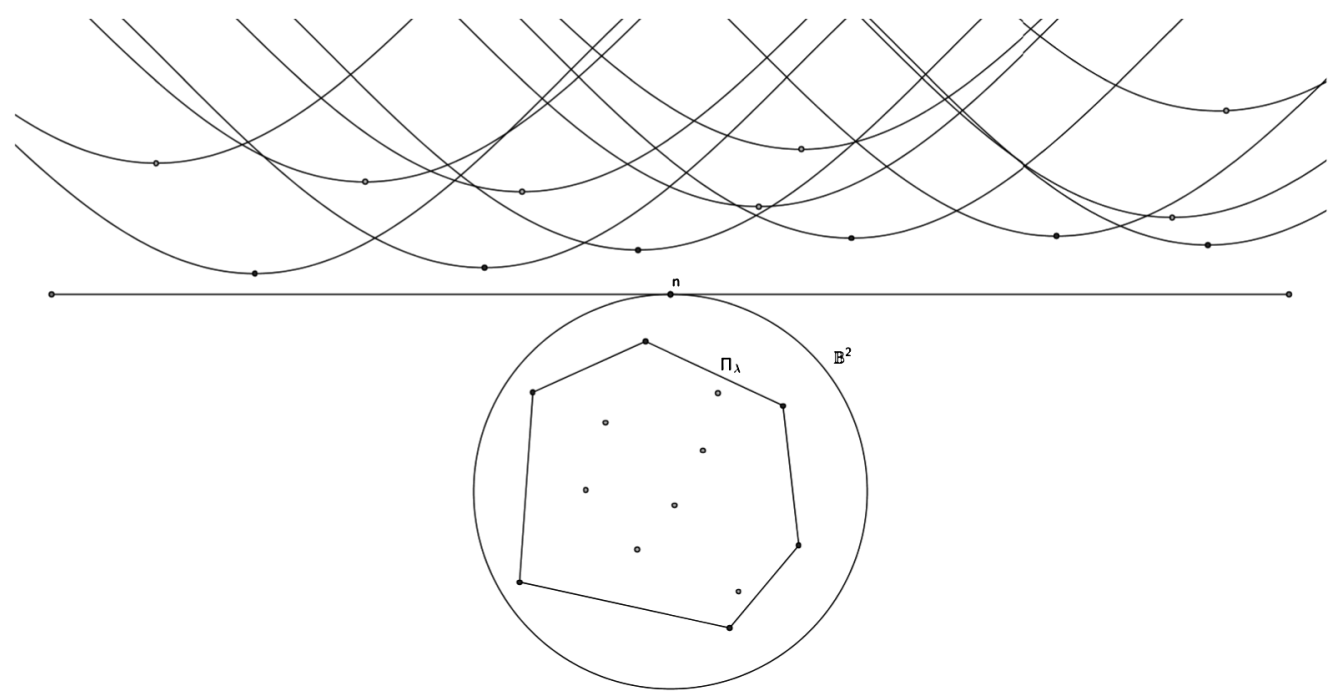

Figure 2. The generalized growth process $\Psi^{(\lambda)}$ associated with the random polytope $\Pi_{\lambda}$.

\subsection{A generalized growth process}

With the rescaled Poisson point process $\eta_{\lambda}^{\mathscr{T}}$ defined in the previous section one can associate what has been called a generalized growth process in [16,41]. We denote by $d_{s}$ the usual geodesic distance on $\mathbb{S}^{d-1}$ and for $x=(v, h) \in \eta_{\lambda}^{\mathscr{T}}$ we define the set

$$
\Pi_{x, \lambda}^{\text {up }}:=\left\{\left(v^{\prime}, h^{\prime}\right) \in \mathbb{R}^{d-1} \times \mathbb{R}_{+}: h^{\prime} \geq \lambda^{\frac{2}{d+1}}\left(1-\cos \omega\left(v^{\prime}, v\right)\right)+h \cos \omega\left(v^{\prime}, v\right)\right\},
$$

where $\omega\left(v^{\prime}, v\right):=d_{s}\left(\exp \left(\lambda^{-\frac{1}{d+1}} v\right), \exp \left(\lambda^{-\frac{1}{d+1}} v^{\prime}\right)\right)$. Following [16,41], the generalized growth process $\Psi^{(\lambda)}$ is now given by

$$
\Psi^{(\lambda)}:=\bigcup_{x \in \eta_{\lambda}^{\mathscr{T}}} \Pi_{x, \lambda}^{\mathrm{up}} .
$$

We say that a particle of $\Psi^{(\lambda)}$ is extreme if it is not completely covered by other particles and we denote the set of extreme points of the extreme particles of $\Psi^{(\lambda)}$ by $\operatorname{ext}\left(\Psi^{(\lambda)}\right)$. In particular, we notice that the image under the scaling transformation $\mathscr{T}_{\lambda}$ of the set of extreme points of the random polytope $\Pi_{\lambda}$ coincides with $\operatorname{ext}\left(\Psi^{(\lambda)}\right)$, see Figure 2 , where all the sets $\Pi_{x, \lambda}^{\mathrm{up}}$ associated with the vertices of $\Pi_{\lambda}$ cover the ones that correspond to the inner points of $\Pi_{\lambda}$. Let us recall from [41], Lemma 3.2, that the probability that a point $x=(v, h) \in \mathcal{R}_{\lambda}$ belongs to $\operatorname{ext}\left(\Psi^{(\lambda)}\right)$ decays exponentially with the height $h$ of $x$. More precisely, we have that there are universal constants $c_{1}, c_{2} \in(0, \infty)$ such that

$$
\mathbb{P}\left(x \in \operatorname{ext}\left(\Psi^{(\lambda)}\right)\right) \leq c_{1} \exp \left(-c_{1}^{-1} h^{(d+1) / 2}\right)
$$

for all $\lambda \geq c_{2}$, uniformly in $x$. 


\section{Main results for Poisson polytopes}

Let $\eta_{\lambda}$ be the restriction to $\mathbb{B}^{d}$ of a stationary Poisson point process in $\mathbb{R}^{d}$ with intensity $\lambda>0$ and let $\Pi_{\lambda}$ be the convex hull of $\eta_{\lambda}$. For a key geometric functional $\xi \in \Xi$ and its rescaled version $\xi^{(\lambda)}$ as considered in Section 2.2, we introduce the spatial empirical measure

$$
\begin{aligned}
\mu_{\lambda}^{\xi} & :=\sum_{x \in \eta_{\lambda}} \xi\left(x, \eta_{\lambda}\right) \delta_{x} \\
& =\sum_{x^{\prime} \in \eta_{\lambda}^{\mathscr{T}}} \xi^{(\lambda)}\left(x^{\prime}, \eta_{\lambda}^{\mathscr{T}}\right) \delta_{\mathscr{T}_{\lambda}^{-1}\left(x^{\prime}\right)},
\end{aligned}
$$

where $\delta_{x}$ stands for the Dirac measure at $x$. The centered version of $\mu_{\lambda}^{\xi}$ is throughout denoted by $\bar{\mu}_{\lambda}^{\xi}:=\mu_{\lambda}^{\xi}-\mathbb{E} \mu_{\lambda}^{\xi}$. In order to ensure non-trivial scaling behaviors, we define the individual scaling exponents

$$
e[\xi]:= \begin{cases}1, & \xi \in\left\{\xi_{V_{1}}, \ldots, \xi_{V_{d}}, \xi_{\mathrm{VF}}\right\}, \\ 0, & \xi=\xi_{f_{j}} \text { for some } j \in\{0, \ldots, d-1\} .\end{cases}
$$

The reason behind this is that for each of the "volume functionals" the scaling transformation involves a dilation of order $\lambda^{1 / d+1}$ in the spatial region and of order $\lambda^{2 / d+1}$ in the height coordinate, which gives $\lambda^{\frac{d-1}{d+1}} \lambda^{\frac{2}{d+1}}=\lambda^{1}$ in total. Since the number of $j$-faces is not influenced by the scaling transformation, no further scaling exponent is needed here. Further, for $f \in \mathcal{B}\left(\mathbb{B}^{d}\right)$ we define the quantity $\sigma_{\lambda}^{\xi}[f]:=\left(\operatorname{var}\left\langle f, \mu_{\lambda}^{\xi}\right\rangle\right)^{1 / 2}$ and emphasize that for each of the key geometric functionals $\xi$ we consider, there exists a constant $c>0$ only depending on $d$ and $\xi$ such that for all $\lambda \geq c$ one has that

$$
\lambda^{e[\xi]} \sigma_{\lambda}^{\xi}[f] \geq C\left\langle f^{2}, \mathcal{H}_{\mathbb{S}^{d-1}}^{d-1}\right\rangle^{\frac{1}{2}} \lambda^{\frac{d-1}{2(d+1)}}
$$

if $f \in \mathcal{C}\left(\mathbb{B}^{d}\right)$ with another constant $C \in(0, \infty)$ that depends only on $d$ and on $\xi$. This follows from the variance considerations in $[5,16,34]$ and we point out that the continuity of $f$ has essentially been used there to derive the lower variance bound (10). For this reason, we also assume continuity of $f$ in our results.

Our first result is a general concentration inequality for integrals with respect to the empirical measure induced by our key geometric functionals; proofs are postponed to Section 5. To present it, we define the individual weights

$$
w[\xi]:= \begin{cases}2, & \xi \in\left\{\xi_{V_{1}}, \ldots, \xi_{V_{d}}, \xi_{\mathrm{VF}}\right\}, \\ 3 j, & \xi=\xi_{f_{j}} \text { for some } j \in\{0, \ldots, d-1\},\end{cases}
$$

of the key geometric functionals that originate from the moment condition in Lemma 5.2 below and that appear in all our findings. Moreover, recall the definition of $\beta(d)$ in (1). 
Theorem 3.1. Let $f \in \mathcal{C}\left(\mathbb{B}^{d}\right), \xi \in \Xi$ and $y \geq 0$. Suppose that $\left\langle f^{2}, \mathcal{H}_{\mathbb{S} d-1}^{d-1}\right\rangle \neq 0$. Then, for $\lambda \geq c_{1}$,

$$
\mathbb{P}\left(\left|\left\langle f, \bar{\mu}_{\lambda}^{\xi}\right\rangle\right| \geq y \sigma_{\lambda}^{\xi}[f]\right) \leq 2 \exp \left(-\frac{1}{4} \min \left\{\frac{y^{2}}{2^{w[\xi]+\beta(d)}}, c_{2} \lambda^{\frac{d-1}{2(d+1)(w[\xi]+\beta(d))}} y^{\frac{1}{w[\xi]+\beta(d)}}\right\}\right)
$$

with constants $c_{1}, c_{2} \in(0, \infty)$ only depending on $d$ and $\xi$, or on $d$, $\xi$ and $f$, respectively.

Theorem 3.1 should be related to the existing results in the literature. In case that $\xi=\xi_{f_{0}}$, $\left\langle 1, \bar{\mu}_{\lambda}^{\xi}\right\rangle$ is the centred vertex number and a detailed discussion has already been presented in the Introduction. The only other result in the literature we are aware of is a concentration inequality in [44] for the volume (and its closely related version in [35]). Its structure is basically the same as that of the corresponding inequality (2) for the vertex number. In particular, this estimate contains a boundary term $p_{\mathrm{NT}}$ which does not depend on $y$ and is valid only for arguments $y$ in a certain range around zero that depends on $\lambda$.

In contrast to Theorem 3.1, for the next results we could not locate counterparts in the existing literature. To the best of our knowledge, Theorem 3.2 as well as the moderate deviation principles in Theorems 3.5 and 3.6 seem to be the first results in this direction in the context of random polytopes. We start with the asymptotic behaviour of deviation probabilities related to the relative error in the central limit theorem. It is readily seen that Theorem 1.2 presented in the Introduction is a special case of the next theorem.

Theorem 3.2. Let $f \in \mathcal{C}\left(\mathbb{B}^{d}\right)$ with $\left\langle f^{2}, \mathcal{H}_{\mathbb{S}^{d-1}}^{d-1}\right\rangle \neq 0$ and $\xi \in \Xi$. For $0 \leq y \leq c_{3} \lambda^{\frac{d-1}{2(d+1)(2 w[\xi]+2 \beta(d)-1)}}$ and $\lambda \geq c_{4}$ one has that

$$
\begin{gathered}
\left|\log \frac{\mathbb{P}\left(\left\langle f, \bar{\mu}_{\lambda}^{\xi}\right\rangle \geq \sigma_{\lambda}^{\xi}[f] y\right)}{1-\Phi(y)}\right| \leq c_{5}\left(1+y^{3}\right) \lambda^{-\frac{d-1}{2(d+1)(2 w[\xi]+2 \beta(d)-1)}} \quad \text { and } \\
\left|\log \frac{\mathbb{P}\left(\left\langle f, \bar{\mu}_{\lambda}^{\xi}\right\rangle \leq-\sigma_{\lambda}^{\xi}[f] y\right)}{\Phi(-y)}\right| \leq c_{5}\left(1+y^{3}\right) \lambda^{-\frac{d-1}{2(d+1)(2 w[\xi]+2 \beta(d)-1)}}
\end{gathered}
$$

with constants $c_{3}, c_{4}, c_{5} \in(0, \infty)$ only depending on $d$, on $\xi$ and on $f$.

Remark 3.3. Our methods also allow to derive precise estimates for the relative error considered in the previous theorem, which involve the so-called Cramér-Petrov series, cf. [37]. To keep the result simple and to avoid heavy notation, we decided to state it here in a form which suppresses higher-order terms of the asymptotic exponential expansion.

Remark 3.4. One can also derive the following Berry-Esseen estimate. For $\xi \in \Xi, f \in \mathcal{C}\left(\mathbb{B}^{d}\right)$ with $\left\langle f^{2}, \mathcal{H}_{\mathbb{S}^{d-1}}^{d-1}\right\rangle \neq 0$ and a standard Gaussian random variable $Z \sim \mathcal{N}(0,1)$ one has that

$$
\sup _{y \in \mathbb{R}}\left|\mathbb{P}\left(\frac{\left\langle f, \bar{\mu}_{\lambda}^{\xi}\right\rangle}{\sigma_{\lambda}^{\xi}[f]} \leq y\right)-\mathbb{P}(Z \leq y)\right| \leq c_{6} \lambda^{-\frac{d-1}{2(d+1)(2 w[\xi]+2 \beta(d)-1)}}
$$

with a constant $c_{6} \in(0, \infty)$ only depending on $\xi$, on $d$ and on $f$. However, the rate of convergence we get is weaker than that obtained in $[16,34]$ using Stein's method. 
After having investigated deviation probabilities, we turn now to a moderate deviation principle in a partial intermediate regime of rescalings between that of a central limit theorem and a law of large numbers. It is clear that Theorem 1.3 in the Introduction is a special case of this result.

Theorem 3.5. Let $f \in \mathcal{C}\left(\mathbb{B}^{d}\right)$ with $\left\langle f^{2}, \mathcal{H}_{\mathbb{S}^{d-1}}^{d-1}\right\rangle \neq 0$ and $\xi \in \Xi$. Further, let $\left(a_{\lambda}\right)_{\lambda>0}$ be such that

$$
\lim _{\lambda \rightarrow \infty} a_{\lambda}=\infty \quad \text { and } \quad \lim _{\lambda \rightarrow \infty} a_{\lambda} \lambda^{-\frac{d-1}{2(d+1)(2 w[\xi]+2 \beta(d)-1)}}=0
$$

Then $\left(\frac{1}{a_{\lambda}} \frac{\left\langle f, \bar{\mu} \bar{\mu}_{\lambda}^{\xi}\right\rangle}{\sigma_{\lambda}^{\xi}[f]}\right)_{\lambda>0}$ satisfies a moderate deviation principle on $\mathbb{R}$ with speed $a_{\lambda}^{2}$ and rate function $I(y)=\frac{y^{2}}{2}$.

Our final aim is to lift the result of Theorem 3.5 to a moderate deviation principle on the level of measures (a so-called level-2 MDP). To do so, we first need to introduce the necessary topological notions. The weak topology on $\mathcal{M}\left(\mathbb{S}^{d-1}\right)$ is generated by the sets $U_{f, x, \delta}:=\left\{\nu \in \mathcal{M}\left(\mathbb{S}^{d-1}\right)\right.$ : $|\langle f, v\rangle-x|<\varepsilon\}$ with $x \in \mathbb{R}, \varepsilon>0$ and $f \in \mathcal{C}\left(\mathbb{S}^{d-1}\right)$, see [18], Chapter 6.2. It is also known from [18] that $\mathcal{M}\left(\mathbb{S}^{d-1}\right)$ supplied with the weak topology is a locally convex, Hausdorff topological vector space whose topological dual is identified with the collection of linear functionals $v \mapsto$ $\langle f, v\rangle, f \in \mathcal{C}\left(\mathbb{S}^{d-1}\right)$.

To present our result, we recall from Theorem 7.1 in [16] that for all $\xi \in \Xi$ there exists a constant $\sigma_{\infty}^{\xi} \in(0, \infty)$ such that

$$
\lim _{\lambda \rightarrow \infty} \lambda^{-\frac{d-1}{d+1}+2 e[\xi]} \operatorname{var}\left\langle f, \mu_{\lambda}^{\xi}\right\rangle=\left(\sigma_{\infty}^{\xi}\right)^{2}\left\langle f^{2}, \mathcal{H}_{\mathbb{S}^{d-1}}^{d-1}\right\rangle
$$

if $f \in \mathcal{C}\left(\mathbb{B}^{d}\right)$ (the strict positivity of $\sigma_{\infty}^{\xi}$ follows from [16], Corollary 7.1, and from [34], Lemma 8).

Theorem 3.6. Let $\xi \in \Xi$ and let $\left(a_{\lambda}\right)_{\lambda>0}$ be such that (13) is satisfied. Then the family $\left(\frac{1}{a_{\lambda}} \frac{\lambda^{e[\xi]} \bar{\mu}_{\lambda}^{\xi}}{\sigma_{\infty}^{\xi} \lambda^{(d-1) /(2(d+1))}}\right)_{\lambda>0}$ satisfies a moderate deviation principle on $\mathcal{M}\left(\mathbb{S}^{d-1}\right)$, supplied with the weak topology, with speed $a_{\lambda}^{2}$ and rate function

$$
I(v)= \begin{cases}\frac{1}{2}\left\langle\varrho^{2}, \mathcal{H}_{\mathbb{S}^{d-1}}^{d-1}\right\rangle, & v \ll \mathcal{H}_{\mathbb{S}^{d-1}}^{d-1} \text { with density } \varrho=\frac{\mathrm{d} \nu}{\mathrm{d} \mathcal{H}_{\mathbb{S}^{d-1}}^{d-1}}, \\ \infty, & \text { otherwise }\end{cases}
$$

Remark 3.7. In Theorems 3.5 and 3.6, we have seen partial MDPs, covering a part of the regime of scalings between the central limit theorem and the law of large numbers; the full range would correspond to all scalings $a_{\lambda}$ with $a_{\lambda} \rightarrow \infty$ and $a_{\lambda} \lambda^{-(d-1) /(2(d+1))} \rightarrow 0$, as $\lambda \rightarrow \infty$. However, following the discussion in [20], we may argue that there are examples of weakly dependent spatial random systems known in the literature that satisfy a MDP with a Gaussian rate function 
only up to some critical regime of rescalings beyond that of the central limit theorem. For this reason, it might well be the case that for at least some of the key geometric functionals of the random polytopes we consider there is no full-range Gaussian MDP.

Remark 3.8. We do not claim that our results are optimal. To improve them using our methods, one would have to optimize the exponent $w[\xi]+\beta(d)$ at $k$ ! appearing in Proposition 5.1 below. However, for us it is not clear, which (optimal) exponent should be expected, even not in special cases.

Remark 3.9. It is a natural question whether our results presented above continue to hold for underlying convex bodies different from $\mathbb{B}^{d}$. The paper [16] establishes variance asymptotics and central limit theorems for the aforementioned key geometric functionals of $\Pi_{\lambda}$. In a later paper [17], the authors show that for some of these functionals the variance asymptotics and central limit theorems can be transferred to the situation in which the unit ball is replaced by a convex body with sufficiently smooth boundary. The proof is involved and highly technical. It seems likely that also some of our results could - with presumably much effort - be transferred using the methods established in [17]. However, to keep the length of the paper within bounds, we have decided to restrict to the prototypical case of the unit ball, which is also needed in the next section.

\section{Applications to Poisson polyhedra}

We are now going to apply the results obtained in the previous section to a class of Poisson polyhedra that arise as cells of a Poisson hyperplane mosaic. To define them, fix a parameter $\alpha \geq 1$ and let $v_{\alpha}$ be the measure on $\mathbb{R}^{d}$ that is given by the relation

$$
\int_{\mathbb{R}^{d}} v_{\alpha}(\mathrm{d} x) f(x)=\int_{\mathbb{R}^{d} \backslash \mathbb{B}^{d}} \mathrm{~d} x\|x\|^{\alpha-d} f(x),
$$

where $f \in \mathcal{B}\left(\mathbb{R}^{d}\right)$ is non-negative. Now, let $\zeta_{\lambda}$ be a Poisson point process on $\mathbb{R}^{d}$ with intensity measure $\lambda v_{\alpha}$ and notice that $\zeta_{\lambda}\left(\mathbb{B}^{d}\right)=0$ with probability one. We associate with $\zeta_{\lambda}$ a family $\zeta_{\lambda}^{H}$ of random hyperplanes in $\mathbb{R}^{d}$ as follows. For $x \in \zeta_{\lambda}$ let $H_{x}$ be the hyperplane with unit normal vector $x /\|x\|$ and distance $\|x\| / 2$ to the origin. By the mapping properties of Poisson point processes, $\zeta_{\lambda}^{H}$ is a Poisson point process on the space of hyperplanes in $\mathbb{R}^{d}$. The random hyperplanes of $\zeta_{\lambda}^{H}$ dissect the space into random polyhedra and the principal object of our investigations is the almost surely bounded random polyhedron $Z_{\lambda}=Z_{\lambda}(\alpha)$ which contains the origin, i.e.,

$$
Z_{\lambda}:=\bigcap_{x \in \zeta_{\lambda}} H_{x}^{+}
$$

where $H_{x}^{+}$denotes the half-space bounded by $H_{x}$ that contains the origin. This parametric family of random polyhedra has attracted considerable interest in recent years because of its connections to high-dimensional convex geometry and to a version of the famous problem of D.G. Kendall asking for the asymptotic geometry of "large" mosaic cells, see [13,14,22,23,27,38]. 
The model includes the following special case that has received particular attention and is well known in the literature, cf. [38], Chapter 10.2. It is concerned with a stationary Poisson-Voronoi mosaic. To define it, let $\eta$ be a stationary Poisson point process in $\mathbb{R}^{d}$ with unit intensity. For each $x \in \eta$ we define the Voronoi cell $v(x, \eta):=\left\{z \in \mathbb{R}^{d}:\|z-x\| \leq\left\|z-x^{\prime}\right\|\right.$ for all $\left.x^{\prime} \in \eta\right\}$. The collection of all Voronoi cells forms the Poisson-Voronoi mosaic. Its typical cell can intuitively be understood as randomly chosen (and then shifted to the origin) from the set of all Voronoi cells, where each cell has the same chance of being selected, independently of size and shape. As a consequence of Slivnyak's theorem for Poisson point processes, it can be identified with

$$
Z^{\mathrm{PV}}:=\left\{z \in \mathbb{R}^{d}:\|z\| \leq\|z-x\| \text { for all } x \in \eta\right\},
$$

that is, as the Voronoi cell of the origin, see [38]. By the inradius $R_{\mathrm{in}}\left(Z^{\mathrm{PV}}\right)$ of $Z^{\mathrm{PV}}$ we understand the radius of the largest ball centred at the origin that is contained in $Z^{\mathrm{PV}}$ and we denote by $Z_{r}^{\mathrm{PV}}$ the typical Poisson-Voronoi cell conditioned on the event that $R_{\text {in }}\left(Z^{\mathrm{PV}}\right) \geq r$ for some $r \geq 1$, rescaled by a factor $r^{-1}$. It is remarkable that its distribution coincides with that of the random polyhedron $Z_{\lambda}$ under the particular choice $\alpha=d$ and $\lambda=(2 r)^{d}$, cf. [13,14]. It is known from these works that

$$
\mathbb{E} f_{j}\left(Z_{r}^{\mathrm{PV}}\right) \sim m_{j} r^{\frac{d(d-1)}{d+1}} \quad \text { and } \quad \operatorname{var} f_{j}\left(Z_{r}^{\mathrm{PV}}\right) \sim v_{j}^{2} r^{\frac{d(d-1)}{d+1}}, \quad j \in\{0, \ldots, d-1\},
$$

with constants $m_{j}, v_{j} \in(0, \infty)$ depending only on $d$ and on $j$, where we write $f(r) \sim g(r)$ for two functions $f, g: \mathbb{R} \rightarrow \mathbb{R}$ if $f(r) / g(r) \rightarrow 1$, as $r \rightarrow \infty$. These relations describe the first- and second-order asymptotic combinatorial complexity of typical Poisson-Voronoi cells with large inradius. Furthermore, qualitative asymptotic normality of $f_{j}\left(Z_{r}^{\mathrm{PV}}\right)$ has also been obtained in $[13,14]$. (The results in these papers are formulated only for the case $j=d-1$ and in [14] even for $d=2$, but the extension to arbitrary $j \in\{0, \ldots, d-1\}$ and $d$ is straight forward.)

We are also interested in the combinatorial structure of the random polyhedra $Z_{\lambda}$ and use the duality between $Z_{\lambda}$ and the random polytopes $\Pi_{\lambda}$ developed in [13,14] to derive a concentration inequality, explicit bounds for the relative error in the central limit theorem, a Berry-Esseen bound as well as a moderate deviation principle for $f_{j}\left(Z_{\lambda}^{\mathrm{PV}}\right)$. This adds to the various known contributions around Kendall's problem, see [13,14,27] and the references cited therein. Since the results we obtain are formally the same as in Section 3 with $\left\langle f, \bar{\mu}_{\lambda}^{\xi}\right\rangle$ there replaced by $f_{j}\left(Z_{\lambda}^{\mathrm{PV}}\right)$, we state them here for the particularly attractive Poisson-Voronoi case $\alpha=d$ and $\lambda=(2 r)^{d}$ only.

Theorem 4.1. Let $j \in\{0, \ldots, d-1\}$.

(i) There are constants $c_{1}, c_{2} \in(0, \infty)$ only depending on $d$ and $j$, such that, for $r \geq$ $\max \left\{c_{1}, 1\right\}$,

$$
\begin{aligned}
& \mathbb{P}\left(\left|r^{-\frac{d(d-1)}{d+1}} f_{j}\left(Z_{r}^{\mathrm{PV}}\right)-m_{j}\right| \geq y v_{j}\right) \\
& \quad \leq 2 \exp \left(-\frac{1}{4} \min \left\{\frac{y^{2}}{2^{3 j+\beta(d)}}, c_{2} r^{\frac{d(d-1)}{2(d+1)(3 j+\beta(d))}} y^{\frac{1}{3 j+\beta(d)}}\right\}\right)
\end{aligned}
$$

for all $y \geq 0$. 
(ii) For $0 \leq y \leq c_{3} r^{\frac{d(d-1)}{2(d+1)(6 j+2 \beta(d)-1)}}$ and $r \geq \max \left\{c_{4}, 1\right\}$ one has that

$$
\begin{aligned}
\left|\log \frac{\mathbb{P}\left(r^{-\frac{d(d-1)}{d+1}} f_{j}\left(Z_{r}^{\mathrm{PV}}\right)-m_{j} \geq y v_{j}\right)}{1-\Phi(y)}\right| & \leq c_{5}\left(1+y^{3}\right) r^{-\frac{d(d-1)}{2(d+1)(6 j+2 \beta(d)-1)}} \quad \text { and } \\
\left|\log \frac{\mathbb{P}\left(r^{-\frac{d(d-1)}{d+1}} f_{j}\left(Z_{r}^{\mathrm{PV}}\right)-m_{j} \leq-y v_{j}\right)}{\Phi(-y)}\right| & \leq c_{5}\left(1+y^{3}\right) r^{-\frac{d(d-1)}{2(d+1)(6 j+2 \beta(d)-1)}}
\end{aligned}
$$

with constants $c_{3}, c_{4}, c_{5} \in(0, \infty)$ only depending on $d$ and on $j$.

(iii) One can find a constant $c_{6} \in(0, \infty)$ only depending on $d$ and $j$ such that

$$
\sup _{y \in \mathbb{R}}\left|\mathbb{P}\left(\frac{f_{j}\left(Z_{r}^{\mathrm{PV}}\right)-m_{j} r^{\frac{d(d-1)}{d+1}}}{v_{j} r^{d(d-1) /(2(d+1))}} \leq y\right)-\mathbb{P}(Z \leq y)\right| \leq c_{6} r^{-\frac{d(d-1)}{2(d+1)(6 j+2 \beta(d)-1)}}
$$

for all sufficiently large $r$, where $Z \sim \mathcal{N}(0,1)$ is a standard Gaussian random variable.

(iv) Suppose that $\left(a_{r}\right)_{r \geq 1}$ satisfies

$$
\lim _{r \rightarrow \infty} a_{r}=\infty \quad \text { and } \quad \lim _{r \rightarrow \infty} a_{r} r^{-\frac{d(d-1)}{2(d+1)(6 j+2 \beta(d)-1)}} .
$$

Then

$$
\left(\frac{1}{a_{r}} \frac{f_{j}\left(Z_{r}^{\mathrm{PV}}\right)-m_{j} r^{\frac{d(d-1)}{d+1}}}{v_{j} r^{d(d-1) /(2(d+1))}}\right)_{r \geq 1}
$$

satisfies a moderate deviation principle on $\mathbb{R}$ with speed $a_{r}^{2}$ and rate function $I(y)=\frac{y^{2}}{2}$.

Proof. The inversion technique introduced in [14] implies that $f_{j}\left(Z_{r}^{\mathrm{PV}}\right)=f_{d-j-1}\left(\Pi_{r}\right)$ almost surely for all $j \in\{0, \ldots, d-1\}$. This way, the result readily follows from Theorems 3.1, 3.2, 3.5 and Remark 3.4 in combination with (14).

\section{Proofs of the main results for Poisson polytopes}

Within this section all constants $\lambda_{0}, c, c_{1}, c_{2}, \ldots$ are strictly positive, finite and such that they only depend on the space dimension $d$ and the key geometric functional $\xi$ belonging to the set $\Xi=\left\{\xi_{\mathrm{VF}}, \xi_{V_{1}}, \ldots, \xi_{V_{d}}, \xi_{f_{0}}, \ldots, \xi_{f_{d-1}}\right\}$ we consider, unless otherwise specified. The constants may change from line to line. Moreover, recall the definition of the individual scaling exponents $e[\xi]$ given in (9).

\subsection{Preparations}

For $k \in \mathbb{N}$ and $\lambda>0$ we introduce the singular differential $\overline{\mathrm{d}}_{\lambda}$ by the relation

$$
\int_{\left(\mathbb{B}^{d}\right)^{k}} \overline{\mathrm{d}}_{\lambda}\left(x_{1}, \ldots, x_{k}\right) g\left(x_{1}, \ldots, x_{k}\right)=\lambda \int_{\mathbb{B}^{d}} \mathrm{~d} x g(x, \ldots, x), \quad g \in \mathcal{B}\left(\left(\mathbb{B}^{d}\right)^{k}\right) .
$$


Furthermore, if $\Sigma_{k}$ denotes the set of unordered partitions of $[k]:=\{1, \ldots, k\}$ and $\Sigma_{k, p}$ the subset of partitions into precisely $p \leq k$ blocks, we put

$$
\tilde{\mathrm{d}}_{\lambda} \mathbf{x}:=\sum_{\sigma \in \Sigma_{k}} \prod_{L \in \sigma} \mathrm{d}_{\lambda} \mathbf{x}_{L}=\sum_{\substack{p \in\{1, \ldots, k\} \\ \sigma \in \Sigma_{k, p}}} \prod_{L \in \sigma} \mathrm{d}_{\lambda} \mathbf{x}_{L},
$$

where $\mathbf{x}=\left(x_{1}, \ldots, x_{k}\right) \in\left(\mathbb{B}^{d}\right)^{k}$ and $\mathbf{x}_{L}=\left(x_{\ell}: \ell \in L\right) \in\left(\mathbb{B}^{d}\right)^{|L|}$ with $|L|$ being the number of elements in block $L$. To enhance readability and to allow for an easier comparison with [20], we abbreviate this by the shorthand notation

$$
\tilde{\mathrm{d}}_{\lambda} \mathbf{x}=\sum_{L_{1}, \ldots, L_{p} \leq[k]} \overline{\mathrm{d}}_{\lambda} \mathbf{x}_{L_{1}} \cdots \overline{\mathrm{d}}_{\lambda} \mathbf{x}_{L_{p}}
$$

Fix a key geometric functional $\xi \in \Xi$ associated with the random polytopes $\Pi_{\lambda}$ and $k \in \mathbb{N}$. The $k$ th moment measure $M_{\lambda}^{k}(\mathrm{~d} \mathbf{x})$ of the rescaled empirical measure $\lambda^{e[\xi]} \mu_{\lambda}^{\xi}$ (recall (8)) is defined as the measure on $\left(\mathbb{B}^{d}\right)^{k}$ which is absolutely continuous with respect to $\tilde{\mathrm{d}}_{\lambda} \mathbf{x}$ and whose density $m_{\lambda}(\mathbf{x})$ is given by

$$
\begin{aligned}
m_{\lambda}(\mathbf{x})=m_{\lambda}\left(x_{1}, \ldots, x_{k}\right) & =\mathbb{E}\left[\prod_{i=1}^{k} \lambda^{e[\xi]} \xi\left(x_{i}, \eta_{\lambda} \cup\left\{x_{1}, \ldots, x_{k}\right\}\right)\right] \\
& =\mathbb{E}\left[\prod_{i=1}^{k} \lambda^{e[\xi]} \xi^{(\lambda)}\left(\mathscr{T}_{\lambda}\left(x_{i}\right), \eta_{\lambda}^{\mathscr{T}} \cup\left\{\mathscr{T}_{\lambda}\left(x_{1}\right), \ldots, \mathscr{T}_{\lambda}\left(x_{k}\right)\right\}\right)\right]
\end{aligned}
$$

where again $\mathbf{x}=\left(x_{1}, \ldots, x_{k}\right) \in\left(\mathbb{B}^{d}\right)^{k}$. This is a consequence of [20], Proposition 3.1, which in turn follows from the multivariate Mecke equation for Poisson point processes.

Closely related to the moment measures $M_{\lambda}^{k}$ are the so-called cumulant measures associated with $\lambda^{e[\xi]} \mu_{\lambda}^{\xi}$. The sequence $\left(c_{\lambda}^{k}\right)_{k \geq 1}$ of these cumulant measures is defined via the well-known relation between moments and cumulants as

$$
c_{\lambda}^{k}:=\sum_{L_{1}, \ldots, L_{p} \leq[k]}(-1)^{p-1}(p-1) ! M_{\lambda}^{\left|L_{1}\right|} \otimes \cdots \otimes M_{\lambda}^{\left|L_{p}\right|},
$$

where $\otimes$ denotes the operation that forms the product measure, see [20], equation (3.11). Note that $c_{\lambda}^{k}$ is a signed measure on the product space $\left(\mathbb{B}^{d}\right)^{k}$.

Following [7,20], we finally define for non-empty disjoint sets $S, T \subseteq \mathbb{N}$ the (semi-) cluster measure $U_{\lambda}^{S, T}$ on $\left(\mathbb{B}^{d}\right)^{|S \cup T|}$ by

$$
U_{\lambda}^{S, T}:=M_{\lambda}^{|S \cup T|}-M_{\lambda}^{|S|} \otimes M_{\lambda}^{|T|} .
$$


These cluster measures appear in the following decomposition of $c_{\lambda}^{k}$ taken from [7,20]. Namely, for a non-trivial partition $\{S, T\} \preceq[k]$ one has that

$$
c_{\lambda}^{k}=\sum_{S^{\prime}, T^{\prime}, K_{1}, \ldots, K_{m} \leq[k]} c\left(S^{\prime}, T^{\prime}, K_{1}, \ldots, K_{m}\right) U_{\lambda}^{S^{\prime}, T^{\prime}} \otimes M_{\lambda}^{\left|K_{1}\right|} \otimes \cdots \otimes M_{\lambda}^{\left|K_{m}\right|},
$$

where $\left\{S^{\prime}, T^{\prime}, K_{1}, \ldots, K_{m}\right\}$ is a partition of $[k]$ with $S^{\prime} \subseteq S$ and $T^{\prime} \subseteq T$. The numerical coefficients $c\left(S^{\prime}, T^{\prime}, K_{1}, \ldots, K_{m}\right)$ in (16) are known to satisfy the estimate

$$
\sum_{S^{\prime}, T^{\prime}, K_{1}, \ldots, K_{m} \leq[k]}\left|c\left(S^{\prime}, T^{\prime}, K_{1}, \ldots, K_{m}\right)\right| \leq 2^{k} k !
$$

and this upper bound is best possible according to Corollary 3.1 and Lemma 3.2 in [20]. The representation (16) together with the estimate (17) are the starting point for the proof of our main results. We emphasize that although the starting point of our proof is the same as for the results in [7] or [20], many of the further details differ significantly because of the different nature of the functionals we consider (see Remarks 5.4 and 5.6 for more detailed explanations).

\subsection{Cumulant estimates}

This section contains the most technical part of the proof of our main theorems. The key result is the following bound for the integrals of a test function with respect to the cumulant measures introduced in the previous section. In fact, this result can somehow be seen as the main contribution of the present text from which all the other theorems follow. Note that the continuity of the test functions is not needed in this part of the proof. It will enter later when Proposition 5.1 is combined with the variance lower bound (10). Recall the definition (11) of the individual weights $w[\xi]$ of the key geometric functionals $\xi \in \Xi$ as well as that of the parameter $\beta(d)$ from (1). Moreover, for $k \in \mathbb{N}$ and $f \in \mathcal{B}\left(\mathbb{B}^{d}\right)$ we let $f^{\otimes k} \in \mathcal{B}\left(\left(\mathbb{B}^{d}\right)^{k}\right)$ be the $k$-fold tensor product given by $f^{\otimes k}\left(x_{1}, \ldots, x_{k}\right)=f\left(x_{1}\right) \cdots f\left(x_{k}\right)$.

Proposition 5.1. Let $\xi \in \Xi, f \in \mathcal{B}\left(\mathbb{B}^{d}\right)$ and $k \in\{3,4, \ldots\}$. Then, for $\lambda \geq c_{1}$,

$$
\left|\left\langle f^{\otimes k}, c_{\lambda}^{k}\right\rangle\right| \leq c_{2} c_{3}^{k}\|f\|_{\infty}^{k} \lambda^{\frac{d-1}{d+1}}(k !)^{w[\xi]+\beta(d)}
$$

with constants $c_{1}, c_{2}, c_{3} \in(0, \infty)$ only depending on $\xi$ and on $d$.

We divide the proof of Proposition 5.1 into a couple of lemmas. To simplify the notation, for the remainder of this section we fix $k \in\{3,4, \ldots\}$.

We need the following lemma that considerably refines the moment condition from [16].

Lemma 5.2. Let $p \geq 1, x_{1}=\left(v_{1}, h_{1}\right), \ldots, x_{p}=\left(v_{p}, h_{p}\right) \in \mathcal{R}_{\lambda}$ and $p u t \delta:=\min _{i, j=1, \ldots, p} \| v_{i}-$ $v_{j} \|$. 
(i) For sufficiently large $\lambda$ one has that

$$
\mathbb{E}\left|\lambda^{e[\xi]} \xi^{(\lambda)}\left(x_{1}, \eta_{\lambda}^{\mathscr{T}} \cup\left\{x_{1}\right\}\right)\right|^{p} \leq c_{1} c_{2}^{p}(p !)^{w[\xi]} \exp \left(-c_{3} h_{1}^{(d+1) / 2}\right)
$$

and

$$
\left.\mathbb{E}\left|\lambda^{e[\xi]} \xi^{(\lambda)}\left(x_{1}, \eta_{\lambda}^{\mathscr{T}} \cup\left\{x_{1}\right\} \cap \operatorname{Cyl}\left(x_{1}, \frac{\delta}{2}\right)\right)\right|^{p} \leq c_{1} c_{2}^{p}(p !)\right)^{w[\xi]} \exp \left(-c_{3} h_{1}^{(d+1) / 2}\right) .
$$

(ii) Moreover, one has that

$$
E_{1}:=\mathbb{E}\left(\prod_{i=1}^{p} \lambda^{e[\xi]} \xi^{(\lambda)}\left(x_{i}, \eta_{\lambda}^{\mathscr{T}} \cup \bigcup_{j=1}^{p}\left\{x_{j}\right\}\right)\right)^{2} \leq c_{1} c_{2}^{p}(p !)^{2 w[\xi]} \prod_{i=1}^{p} \exp \left(-c_{3} \frac{h_{i}^{(d+1) / 2}}{p}\right)
$$

and

$$
\begin{aligned}
E_{2} & :=\mathbb{E}\left(\prod_{i=1}^{p} \lambda^{e[\xi]} \xi^{(\lambda)}\left(x_{i},\left(\eta_{\lambda}^{\mathscr{T}} \cup \bigcup_{j=1}^{p}\left\{x_{j}\right\}\right) \cap \operatorname{Cyl}\left(x_{i}, \frac{\delta}{2}\right)\right)\right)^{2} \\
& \leq c_{1} c_{2}^{p}(p !)^{2 w[\xi]} \prod_{i=1}^{p} \exp \left(-c_{3} \frac{h_{i}^{(d+1) / 2}}{p}\right)
\end{aligned}
$$

again for sufficiently large $\lambda$.

Proof. We start with part (i) and we only proof the first inequality, the second one follows by the same reasoning. Consider the missed-volume functional $\xi=\xi_{V_{d}}$. We can assume that $x_{1}$ is an extreme point of $\Psi^{(\lambda)}$, since otherwise $\xi^{(\lambda)}\left(x_{1}, \eta_{\lambda}^{\mathscr{T}} \cup\left\{x_{1}\right\}\right)$ is zero. Now, we notice that in this case and for sufficiently large $\lambda$ the random variable $\lambda^{e[\xi]} \xi^{(\lambda)}\left(x_{1}, \eta_{\lambda}^{\mathscr{T}} \cup\left\{x_{1}\right\}\right)$ is bounded by $R\left(x_{1}\right)^{d-1} S\left(x_{1}\right)$, the volume of a cylinder with height $S\left(x_{1}\right)$ whose base is a $(d-1)$-dimensional ball with radius $R\left(x_{1}\right)$. Here, $S\left(x_{1}\right)=\sup _{w \in \mathbb{B}^{d-1}\left(v_{1}, R\left(x_{1}\right)\right)} \partial \Psi^{(\lambda)}(w)$ and $R\left(x_{1}\right)$ stands for the radius of localization of $\xi^{(\lambda)}$ at $x_{1}$. Using (6) and (a simplified version of) [16], equation (4.5), we conclude that, for sufficiently large $\lambda$,

$$
\begin{aligned}
& \mathbb{E}\left|\lambda^{e[\xi]} \xi^{(\lambda)}\left(x_{1}, \eta_{\lambda}^{\mathscr{T}} \cup\left\{x_{1}\right\}\right)\right|^{p} \\
& \quad=p \int_{0}^{\infty} \mathrm{d} s s^{p-1} \mathbb{P}\left(\lambda^{e[\xi]} \xi^{(\lambda)}\left(x_{1}, \eta_{\lambda}^{\mathscr{T}} \cup\left\{x_{1}\right\}\right) \geq s\right) \\
& \quad=p \int_{0}^{\infty} \mathrm{d} s s^{p-1} \mathbb{P}\left(S\left(x_{1}\right) \geq s / R\left(x_{1}\right)^{d-1}\right) \\
& \quad \leq p c_{1} \int_{0}^{\infty} \mathrm{d} r \int_{0}^{\infty} \mathrm{d} s s^{p-1} \exp \left(-c_{2}\left(s / r^{d-1}\right)\right) \exp \left(-c_{3} r^{d+1}\right) \\
& \quad \leq p c_{4} c_{5}^{p} \int_{0}^{\infty} u^{p-1} \exp (-u) \mathrm{d} u \int_{0}^{\infty} v^{(p(d-1)-d) /(d+1)} \exp (-v) \mathrm{d} v \leq c_{6} c_{7}^{p}(p !)^{2} .
\end{aligned}
$$


From the inequalities $\xi_{V_{1}}, \ldots, \xi_{V_{d-1}} \leq \xi_{V_{d}}$ and $\xi_{\mathrm{VF}} \leq \xi_{V_{d}}$ (see [16]), we obtain the result of part (i), up to now without the exponential term, for $\xi_{V_{1}}, \ldots, \xi_{V_{d-1}}$ and $\xi_{\mathrm{VF}}$.

Now, let $\xi=\xi_{f_{j}}$ for some $j \in\{0, \ldots, d-1\}$ and $x_{1} \in \mathcal{R}_{\lambda}$. Moreover, put $R\left(x_{1}\right)$ and $S\left(x_{1}\right)$ as above. By $N$ we denote the number of extreme points of $\Psi^{(\lambda)}$ in $\operatorname{Cyl}\left(x_{1}, R\left(x_{1}\right)\right)$ and observe that the number of $j$-dimensional faces meeting at $x_{1}$ is bounded by $\frac{1}{j+1}\left(\begin{array}{c}N \\ j\end{array}\right) \leq N^{j}$. So,

$$
\mathbb{E}\left|\xi_{f_{j}}^{(\lambda)}\left(x_{1}, \eta_{\lambda}^{\mathscr{T}} \cup\left\{x_{1}\right\}\right)\right|^{p} \leq \mathbb{E} N^{j p}
$$

Writing $v_{\lambda}$ for the intensity measure of the rescaled Poisson point process $\eta_{\lambda}^{\mathscr{T}}$, one easily obtains from (5) that for all $r \in\left[0, \pi \lambda^{1 /(d+1)}\right], \ell \in\left[0, \lambda^{2 /(d+1)}\right]$ and sufficiently large $\lambda, v_{\lambda}\left(\mathrm{Cyl}\left(x_{1}, r\right) \cap\right.$ $(0, \ell)) \leq c_{1} r^{d-1} \ell$, thus

$$
\begin{aligned}
\mathbb{E}\left[N^{p j}\right] \leq & \mathbb{E}\left|\eta_{\lambda}^{\mathscr{T}} \cap \operatorname{Cyl}\left(x_{1}, R\left(x_{1}\right)\right) \cap\left(0, S\left(x_{1}\right)\right)\right|^{p j} \\
\leq & \sum_{i=0}^{\infty} \sum_{m=0}^{\infty} \mathbb{E}\left[P\left(v_{\lambda}\left(\operatorname{Cyl}\left(x_{1}, i+1\right) \cap(0, m+1)\right)\right)^{p j}\right. \\
& \left.\times \mathbf{1}\left(i \leq R\left(x_{1}\right)<i+1, m \leq S\left(x_{1}\right)<m+1\right)\right] \\
\leq & \sum_{i=0}^{\infty} \sum_{m=0}^{\infty} \mathbb{E}\left[P\left(c_{1}(i+1)^{d-1}(m+1)\right)^{p j} \mathbf{1}\left(R\left(x_{1}\right) \geq i, S\left(x_{1}\right) \geq m\right)\right] \\
\leq & \sum_{i=0}^{\infty} \sum_{m=0}^{\infty}\left(\mathbb{E}\left[P\left(c_{1}(i+1)^{d-1}(m+1)\right)^{3 p j}\right]\right)^{1 / 3} \\
& \times \mathbb{P}\left(R\left(x_{1}\right) \geq i\right)^{1 / 3} \mathbb{P}\left(S\left(x_{1}\right) \geq m\right)^{1 / 3},
\end{aligned}
$$

where $P(\alpha) \sim \operatorname{Po}(\alpha)$ denotes a Poisson random variable with mean $\alpha>0$. Well known moment bounds for Poisson random variables and the fact that $(3 p j) ! \leq 9^{p j}((p j) !)^{3}$ imply

$$
\begin{aligned}
\left(\mathbb{E}\left[P\left(c_{1}(i+1)^{d-1}(m+1)\right)^{3 p j}\right]\right)^{1 / 3} & \leq\left(\left(c_{2}(i+1)^{d-1}(m+1)\right)^{3 p j}(3 p j) !\right)^{1 / 3} \\
& \leq c_{3}^{p}(i+1)^{p(d-1) j}(m+1)^{p j}(p j) !
\end{aligned}
$$

Using this together with (6) and [16], equation (4.5), shows that, for sufficiently large $\lambda$,

$$
\begin{aligned}
\mathbb{E} N^{p j} & \leq c_{1} c_{2}^{p}(p j) ! \sum_{i=0}^{\infty}(i+1)^{p(d-1) j} \exp \left(-c_{3} i^{d+1}\right) \sum_{m=0}^{\infty}(m+1)^{p j} \exp \left(-c_{4} m\right) \\
& \leq c_{5} c_{6}^{p}((p j) !)^{3} \leq c_{7} c_{8}^{p}(p !)^{3 j}
\end{aligned}
$$

where we additionally used the elementary inequality $(p j) ! \leq\left(j^{j}\right)^{p}(p !)^{j}$.

We now add the exponential term to the bounds. We notice that $\xi^{(\lambda)}$ is different from zero if and only if $x_{1}$ is an extreme point of $\Psi^{(\lambda)}$. Thus, using the Cauchy-Schwarz inequality and the 
exponential decay property (7) we find that, for $\xi \in\left\{\xi_{V_{1}}, \ldots, \xi_{V_{d}}, \xi_{\mathrm{VF}}\right\}$,

$$
\begin{aligned}
& \left|\mathbb{E}\left(\lambda^{e[\xi]} \xi^{(\lambda)}\left(x_{1}, \eta_{\lambda}^{\mathscr{T}} \cup\left\{x_{1}\right\}\right)\right)^{k}\right| \\
& \quad=\left|\mathbb{E}\left(\lambda^{e[\xi]} \xi^{(\lambda)}\left(x_{1}, \eta_{\lambda}^{\mathscr{T}} \cup\left\{x_{1}\right\}\right) \mathbf{1}\left(x_{1} \in \operatorname{ext}\left(\Psi^{(\lambda)}\right)\right)\right)^{k}\right| \\
& \quad \leq c_{1} c_{2}^{k}(2 k) ! \exp \left(-c_{3} h_{1}^{(d+1) / 2}\right) \leq c_{4} c_{5}^{k}(k !)^{2} \exp \left(-c_{6} h_{1}^{(d+1) / 2}\right)
\end{aligned}
$$

for sufficiently large $\lambda$, since $(2 k) ! \leq 4^{k}(k !)^{2}$. Here, $h_{1}$ is the height coordinate of $x_{1}$. Similarly, using the first bound in the case of the face-numbers with $\xi=\xi_{f_{j}}$ for some $j \in\{0, \ldots, d-1\}$ we find that

$$
\left|\mathbb{E}\left(\lambda^{e[\xi]} \xi^{(\lambda)}\left(x_{1}, \eta_{\lambda}^{\mathscr{T}} \cup\left\{x_{1}\right\}\right)\right)^{k}\right| \leq c_{1} c_{2}^{k}(k !)^{3 j} \exp \left(-c_{3} h_{1}^{(d+1) / 2}\right)
$$

and thus

$$
\left|\mathbb{E}\left(\lambda^{e[\xi]} \xi^{(\lambda)}\left(x_{1}, \eta_{\lambda}^{\mathscr{T}} \cup\left\{x_{1}\right\}\right)\right)^{k}\right| \leq c_{1} c_{2}^{k}(k !)^{w[\xi]} \exp \left(-c_{3} h_{1}^{(d+1) / 2}\right)
$$

for all $\xi \in \Xi$.

For part (ii), we first have from the proof of Lemma 3.2 in [41] and Hölder's inequality that $E_{1}$ is bounded from above by

$$
\prod_{i=1}^{p}\left(\mathbb{E}\left(\lambda^{e[\xi]} \xi^{(\lambda)}\left(x_{i}, \eta_{\lambda}^{\mathscr{T}} \cup\left\{x_{i}\right\}\right)\right)^{2 p}\right)^{1 / p}
$$

For sufficiently large $\lambda$ this can now be estimated by means of part (i) and the result then follows from the fact that $(2 p) ! \leq 4^{p}(p !)^{2}$. The proof for $E_{2}$ is similar by using the second inequality of part (i).

Our next result formalizes the intuition that the cluster measures $U_{\lambda}^{S, T}$ capture the spatial correlations of the rescaled key-geometric functionals. In particular, we show that these correlations decay exponentially fast.

Lemma 5.3. Let $\{S, T\}$ be a non-trivial partition of $[k]$ and $\xi \in \Xi$ be a key geometric functional. Then, for $x_{1}=\left(v_{1}, h_{1}\right), \ldots, x_{k}=\left(v_{k}, h_{k}\right) \in \mathcal{R}_{\lambda}$ and $\lambda \geq c_{1}$ one has that

$$
\left.\left|m_{\lambda}\left(\mathbf{x}_{S \cup T}\right)-m_{\lambda}\left(\mathbf{x}_{S}\right) m_{\lambda}\left(\mathbf{x}_{T}\right)\right| \leq c_{2} c_{3}^{k} k(k !)\right)^{w[\xi]} \exp \left(-c_{3} \delta^{d+1}\right) \prod_{i=1}^{k} \exp \left(-c_{4} \frac{h_{i}^{(d+1) / 2}}{k}\right) .
$$

Here, $\delta:=\min _{s \in S, t \in T}\left\|v_{s}-v_{t}\right\|$ is the separation of the points in $\mathbb{R}^{d-1}$, and

$$
m_{\lambda}\left(\mathbf{x}_{S}\right):=\mathbb{E}\left[\prod_{s \in S} \lambda^{e[\xi]} \xi^{(\lambda)}\left(x_{S}, \eta_{\lambda}^{\mathscr{T}} \cup \bigcup_{s^{\prime} \in S}\left\{x_{s^{\prime}}\right\}\right)\right]
$$

with $m_{\lambda}\left(\mathbf{x}_{T}\right)$ and $m_{\lambda}\left(\mathbf{x}_{S \cup T}\right)$ defined similarly. 
Proof. Define the random variables

$$
\begin{aligned}
X & :=\prod_{s \in S} \lambda^{e[\xi]} \xi^{(\lambda)}\left(x_{s}, \eta_{\lambda}^{\mathscr{T}} \cup \bigcup_{s^{\prime} \in S}\left\{x_{s^{\prime}}\right\}\right) \text { and } \\
X_{\delta} & :=\prod_{s \in S} \lambda^{e[\xi]} \xi^{(\lambda)}\left(x_{s},\left(\eta_{\lambda}^{\mathscr{T}} \cup \bigcup_{s^{\prime} \in S}\left\{x_{s^{\prime}}\right\}\right) \cap \operatorname{Cyl}\left(x_{s}, \frac{\delta}{2}\right)\right),
\end{aligned}
$$

where, recall, for a point $x=(v, h), \operatorname{Cyl}(x, r)$ stands for the cylinder $\mathbb{B}^{d-1}(v, r) \times \mathbb{R}_{+}$. Similarly, the random variables $Y, Y_{\delta}, Z$ and $Z_{\delta}$ are defined, where instead of the set $S$ the sets $T$ and $S \cup T$ are used in the definition, respectively. Since for $s \in S$ and $t \in T, \operatorname{Cyl}\left(x_{s}, \frac{\delta}{2}\right) \cap \operatorname{Cyl}\left(x_{t}, \frac{\delta}{2}\right)=\varnothing$ by definition of the separation $\delta$ we have, by independence, that $\mathbb{E} W_{\delta}=\mathbb{E} X_{\delta} \mathbb{E} Y_{\delta}$ and hence

$$
\begin{aligned}
& m_{\lambda}\left(\mathbf{x}_{S \cup T}\right)-m_{\lambda}\left(\mathbf{x}_{S}\right) m_{\lambda}\left(\mathbf{x}_{T}\right) \\
& \quad=\mathbb{E} W-\mathbb{E} X \mathbb{E} Y \\
& \quad=\mathbb{E} W_{\delta}-\mathbb{E} X_{\delta} \mathbb{E} Y_{\delta}+\mathbb{E}\left(W-W_{\delta}\right)-\mathbb{E} X_{\delta} \mathbb{E}\left(Y-Y_{\delta}\right)-\mathbb{E} Y \mathbb{E}\left(X-X_{\delta}\right) \\
& \quad=\mathbb{E}\left(W-W_{\delta}\right)-\mathbb{E} X_{\delta} \mathbb{E}\left(Y-Y_{\delta}\right)-\mathbb{E} Y \mathbb{E}\left(X-X_{\delta}\right) .
\end{aligned}
$$

Let $N_{S}$ denote the event that the radius of localization of at least one $x_{s}$ with $s \in S$ exceeds $\delta / 2$. We obtain from Hölder's inequality that

$$
\mathbb{E}\left|X-X_{\delta}\right|=\mathbb{E}\left|X-X_{\delta} \mathbf{1}_{N_{S}^{c}}-X_{\delta} \mathbf{1}_{N_{S}}\right|=\mathbb{E}\left|X_{\delta} \mathbf{1}_{N_{S}}\right| \leq\left(\mathbb{E} X_{\delta}^{2}\right)^{1 / 2} \mathbb{P}\left(N_{S}\right)^{1 / 2}
$$

since on the complement of $N_{S}$ we clearly have that $X$ coincides with $X_{\delta}$. The moment in the first factor is bounded by

$$
c_{1}^{|S|}((|S|) !)^{2 w[\xi]} \prod_{s \in S} \exp \left(-c_{2} \frac{h_{s}^{(d+1) / 2}}{|S|}\right)
$$

by Lemma 5.2 and the probability is bounded by $c_{1}|S| \exp \left(-c_{2} \delta^{d+1}\right)$ in view of the exponential localization property (6). Thus,

$$
\begin{aligned}
\mathbb{E}\left|X-X_{\delta}\right| & \leq c_{1} c_{2}^{k} \sqrt{|S|}((|S|) !)^{w[\xi]} \exp \left(-c_{3} \delta^{d+1}\right) \prod_{s \in S} \exp \left(-c_{4} \frac{h_{s}^{(d+1) / 2}}{|S|}\right) \\
& \leq c_{5} c_{6}^{k} k((|S|) !)^{w[\xi]} \exp \left(-c_{7} \delta^{d+1}\right) \prod_{s \in S} \exp \left(-c_{8} \frac{h_{s}^{(d+1) / 2}}{k}\right)
\end{aligned}
$$

with a similar estimate also for $\mathbb{E}\left|Y-Y_{\delta}\right|$ and $\mathbb{E}\left|W-W_{\delta}\right|$, since $|S|,|T|,|S \cup T| \leq k$.

On the other hand, Lemma 5.2 also yields that

$$
\mathbb{E}\left|X_{\delta}\right| \leq c_{1} c_{2}^{k}((|S|) !)^{w[\xi]} \prod_{s \in S} \exp \left(-c_{3} \frac{h_{s}^{(d+1) / 2}}{k}\right)
$$


and

$$
\mathbb{E}|Y| \leq c_{1} c_{2}^{k}((|T|) !)^{w[\xi]} \prod_{t \in T} \exp \left(-c_{3} \frac{h_{t}^{(d+1) / 2}}{k}\right) .
$$

Combining the above estimates with $(|S|) !(|T|) ! \leq k !$ completes the proof.

Remark 5.4. Lemma 5.3 is a modification of Lemma 5.2 in [7] or Lemma 3.3 in [20], which exhibits a characteristic feature of random polytopes that is not present in the aforementioned papers. In particular, Lemma 5.3 shows that, in the rescaled picture, only points close to the (tangent) hyperplane $\mathbb{R}^{d-1}$ contribute to $\mu_{\lambda}^{\xi}$, while points with a large height coordinate can asymptotically be neglected.

We define the diagonal $\Delta:=\left\{(x, \ldots, x) \in\left(\mathbb{B}^{d}\right)^{k}: x \in \mathbb{B}^{d}\right\}$ and for $\mathbf{x} \in\left(\mathbb{B}^{d}\right)^{k}$ with $\mathbf{x}=$ $\left(x_{1}, \ldots, x_{k}\right)$ and $\mathscr{T}_{\lambda}\left(x_{1}\right)=\left(v_{1}, h_{1}\right), \ldots, \mathscr{T}_{\lambda}\left(x_{k}\right)=\left(v_{k}, h_{k}\right) \in \mathcal{R}_{\lambda}$ we put

$$
\delta(\mathbf{x}):=\delta\left(x_{1}, \ldots, x_{k}\right):=\max \left\{d\left(v_{S}, v_{T}\right):\{S, T\} \preceq[k]\right\},
$$

where $d\left(v_{S}, v_{T}\right):=\min \left\{\left\|v_{s}-v_{t}\right\|: s \in S, t \in T\right\}$. We now observe that $\left(\mathbb{B}^{d}\right)^{k} \backslash \Delta$ can be written as a disjoint union of sets $\sigma(\{S, T\}) \subseteq\left(\mathbb{B}^{d}\right)^{k}$ with non-trivial partitions $\{S, T\} \preceq[k]$ in such a way that $\delta(\mathbf{x})=d\left(v_{S}, v_{T}\right)$ for all $\mathbf{x} \in \sigma(\{S, T\})$. Loosely speaking, this means that we choose for each $\mathbf{x} \in\left(\mathbb{B}^{d}\right)^{k} \backslash \Delta$ exactly that partition $\{S, T\}$ that fulfils $\delta(\mathbf{x})=d\left(v_{S}, v_{T}\right)$. As a consequence, $\left\langle f^{\otimes k}, c_{\lambda}^{k}\right\rangle$ can be decomposed as follows:

$$
\left\langle f^{\otimes k}, c_{\lambda}^{k}\right\rangle=\int_{\Delta} \mathrm{d} c_{\lambda}^{k} f^{\otimes k}+\sum_{S, T \leq[k]} \int_{\sigma(\{S, T\})} \mathrm{d} c_{\lambda}^{k} f^{\otimes k} .
$$

We consider both terms in (21) separately and start with the diagonal term.

Lemma 5.5. For $\xi \in \Xi$ and $f \in \mathcal{B}\left(\mathbb{B}^{d}\right)$ one has that

$$
\left|\int_{\Delta} \mathrm{d} c_{\lambda}^{k} f^{\otimes k}\right| \leq c_{1} c_{2}^{k}(k !)^{w[\xi]}\|f\|_{\infty}^{k} \lambda^{\frac{d-1}{d+1}}
$$

for all $\lambda \geq c_{3}$.

Proof. By definition (15) of the cumulant measures we have that

$$
\begin{aligned}
\int_{\Delta} \mathrm{d} c_{\lambda}^{k} f^{\otimes k} & =\sum_{L_{1}, \ldots, L_{p} \leq[k]}(-1)^{(p-1)}(p-1) ! \int_{\Delta} \mathrm{d}\left(M_{\lambda}^{\left|L_{1}\right|} \otimes \cdots \otimes M_{\lambda}^{\left|L_{p}\right|}\right) f^{\otimes k} \\
& =\sum_{L_{1}, \ldots, L_{p} \leq[k]}(-1)^{(p-1)}(p-1) ! \int_{\Delta} \tilde{\mathrm{d}}_{\lambda} \mathbf{x}_{L_{1}} \cdots \tilde{\mathrm{d}}_{\lambda} \mathbf{x}_{L_{p}} m_{\lambda}\left(\mathbf{x}_{L_{1}}\right) \cdots m_{\lambda}\left(\mathbf{x}_{L_{p}}\right) f^{\otimes k}(\mathbf{x}) .
\end{aligned}
$$


Since we are integrating over the diagonal $\Delta, \mathbf{x}$ is of the form $(x, \ldots, x)$ for some $x \in \mathbb{B}^{d}$ and we can only have $p=1$ in the above sum. We thus have that

$$
\begin{aligned}
& \left|\int_{\Delta} \mathrm{d} c_{\lambda}^{k} f^{\otimes k}\right| \\
& \quad \leq\|f\|_{\infty}^{k} \lambda \int_{\mathbb{B}^{d}} \mathrm{~d} x\left|\mathbb{E}\left(\lambda^{e[\xi]} \xi^{(\lambda)}\left(\mathscr{T}_{\lambda}(x), \eta_{\lambda}^{\mathscr{T}} \cup\left\{\mathscr{T}_{\lambda}(x)\right\}\right)\right)^{k}\right| \\
& \quad \leq c_{1} c_{2}^{k}\|f\|_{\infty}^{k} \lambda(k !)^{w[\xi]} \int_{\mathbb{B}^{d}} \mathrm{~d} x \exp \left(-c_{3} h^{(d+1) / 2}\right)
\end{aligned}
$$

in view of Lemma 5.2, where again $h$ is the height coordinate of $x$ under the transformation $\mathscr{T}_{\lambda}$. Integrating this expression over $\mathbb{B}^{d}$ by introducing spherical coordinates and taking into account the definition (4) of $\mathscr{T}_{\lambda}$ yields that

$$
\begin{aligned}
\left|\int_{\Delta} \mathrm{d} c_{\lambda}^{k} f^{\otimes k}\right| \leq & c_{1} c_{2}^{k}(k !)^{w[\xi]}\|f\|_{\infty}^{k} \lambda \int_{\mathbb{S}^{d-1}} \mathcal{H}_{\mathbb{S}^{d-1}}^{d-1}(\mathrm{~d} u) \\
& \times \int_{0}^{\lambda \frac{2}{d+1}} \mathrm{~d} h \exp \left(-c_{3} h^{(d+1) / 2}\right) \lambda^{-\frac{2}{d+1}}\left(1-\lambda^{-\frac{2}{d+1}} h\right)^{d-1} \\
\leq & c_{4} c_{5}^{k}(k !)^{w[\xi]}\|f\|_{\infty}^{k} \lambda^{1-\frac{2}{d+1}} \int_{\mathbb{S}^{d-1}} \mathcal{H}_{\mathbb{S}^{d-1}}^{d-1}(\mathrm{~d} u) \int_{0}^{\infty} \mathrm{d} h \exp \left(-c_{6} h^{(d+1) / 2}\right) .
\end{aligned}
$$

Using now the fact that $1-\frac{2}{d+1}=\frac{d-1}{d+1}, \int_{\mathbb{S}^{d-1}} \mathcal{H}_{\mathbb{S}^{d-1}}^{d-1}(\mathrm{~d} u)=d \kappa_{d}$ and that

$$
\int_{0}^{\infty} \mathrm{d} h \exp \left(-c_{1} h^{(d+1) / 2}\right)=c_{2},
$$

we conclude that

$$
\left|\int_{\Delta} \mathrm{d} c_{\lambda}^{k} f^{\otimes k}\right| \leq c_{1} c_{2}^{k}(k !)^{w[\xi]}\|f\|_{\infty}^{k} \lambda^{\frac{d-1}{d+1}}
$$

for sufficiently large $\lambda$. This completes the proof.

Remark 5.6. We would like to point out that the proof for the diagonal term we presented is a modification of that in [20]. It incorporates the moment bounds established in Lemma 5.2 and carefully deals with the new effect that only points from $\eta_{\lambda}$ close to the boundary of $\mathbb{B}^{d}$ contribute to the geometry of the random polytopes as well as with the specific geometric situation induced by the scaling transformation $\mathscr{T}_{\lambda}$. Such a modification is no longer possible for large parts of the proof dealing with the off-diagonal term we treat next.

In a next step, we derive a first upper bound of the off-diagonal term in (21). 
Lemma 5.7. Let $\xi \in \Xi$ and $f \in \mathcal{B}\left(\mathbb{B}^{d}\right)$. Then, for $\lambda \geq c_{1}$,

$$
\begin{aligned}
& \left|\sum_{S, T \leq[k]} \int_{\sigma(\{S, T\})} \mathrm{d} c_{\lambda}^{k} f^{\otimes k}\right| \\
& \leq c_{2} c_{3}^{k} k(k !)^{w[\xi]+1}\|f\|_{\infty}^{k} \lambda^{\frac{d-1}{d+1}} \sum_{L_{1}, \ldots, L_{p} \leq[k]} \int_{0}^{\infty} \mathrm{d} h_{1} \cdots \int_{0}^{\infty} \mathrm{d} h_{p} \\
& \quad \times \int_{\left(\mathbb{R}^{d-1}\right)^{p-1}} \mathrm{~d} \mathbf{v} \exp \left(-c_{4} \delta(0, \mathbf{v})^{d+1}\right) \prod_{i=1}^{p} \exp \left(-c_{5} \frac{h_{i}^{(d+1) / 2}}{k}\right) .
\end{aligned}
$$

Proof. We combine (16) with the definition of the singular differential to see that

$$
\begin{aligned}
\int_{\sigma(\{S, T\})} \mathrm{d} c_{\lambda}^{k} f^{\otimes k}= & \sum_{S^{\prime}, T^{\prime}, K_{1}, \ldots, K_{m} \leq[k]} c\left(S^{\prime}, T^{\prime}, K_{1}, \ldots, K_{m}\right) \int_{\sigma(\{S, T\})} \tilde{\mathrm{d}}_{\lambda} \mathbf{x} f(\mathbf{x}) \\
& \times\left(m_{\lambda}\left(\mathbf{x}_{S^{\prime} \cup T^{\prime}}\right)-m_{\lambda}\left(\mathbf{x}_{S^{\prime}}\right) m_{\lambda}\left(\mathbf{x}_{T^{\prime}}\right)\right) m_{\lambda}\left(\mathbf{x}_{K_{1}}\right) \cdots m_{\lambda}\left(\mathbf{x}_{K_{m}}\right),
\end{aligned}
$$

where we also used that for each set $L \in\left\{S^{\prime}, T^{\prime}, S^{\prime} \cup T^{\prime}, K_{1}, \ldots, K_{m}\right\}, m_{\lambda}\left(\mathbf{x}_{L}\right)$ is the density of the moment measure $M_{\lambda}^{|L|}$. Now, Lemma 5.3 shows that

$$
\begin{aligned}
& \left|m_{\lambda}\left(\mathbf{x}_{S^{\prime} \cup T^{\prime}}\right)-m_{\lambda}\left(\mathbf{x}_{S^{\prime}}\right) m_{\lambda}\left(\mathbf{x}_{T^{\prime}}\right)\right| \\
& \quad \leq c_{1} c_{2}^{k} k\left(\left|S^{\prime} \cup T^{\prime}\right| !\right)^{w[\xi]} \exp \left(-c_{3} d\left(v_{S^{\prime}}, v_{T^{\prime}}\right)^{d+1}\right) \prod_{r \in S^{\prime} \cup T^{\prime}} \exp \left(-c_{4} \frac{h_{r}^{(d+1) / 2}}{k}\right),
\end{aligned}
$$

where, as usual, $\mathscr{T}_{\lambda}\left(x_{1}\right)=\left(v_{1}, h_{1}\right), \ldots, \mathscr{T}_{\lambda}\left(x_{k}\right)=\left(v_{k}, h_{k}\right)$. Furthermore, we get from the moment bounds stated in Lemma 5.2 that

$$
\left|m_{\lambda}\left(\mathbf{x}_{K_{i}}\right)\right| \leq c_{1} c_{2}^{\left|K_{i}\right|}\left(\left|K_{i}\right| !\right)^{w[\xi]} \prod_{i \in K_{i}} \exp \left(-c_{3} \frac{h_{i}^{(d+1) / 2}}{k}\right) .
$$

Next, since $S^{\prime} \subseteq S$ and $T^{\prime} \subseteq T$, we necessarily have that $d\left(v_{S^{\prime}}, v_{T^{\prime}}\right) \geq d\left(v_{S}, v_{T}\right)$ and thus

$$
\begin{gathered}
\left|\left(m_{\lambda}\left(\mathbf{x}_{S^{\prime} \cup T^{\prime}}\right)-m_{\lambda}\left(\mathbf{x}_{S^{\prime}}\right) m_{\lambda}\left(\mathbf{x}_{T^{\prime}}\right)\right) m_{\lambda}\left(\mathbf{x}_{K_{1}}\right) \cdots m_{\lambda}\left(\mathbf{x}_{K_{m}}\right)\right| \\
\leq c_{1} c_{2}^{k} k\left(\left|S^{\prime} \cup T^{\prime}\right| !\right)^{w[\xi]}\left(\left|K_{1}\right| !\right)^{w[\xi]} \cdots\left(\left|K_{m}\right| !\right)^{w[\xi]} \\
\quad \times \exp \left(-c_{3} d\left(v_{S}, v_{T}\right)^{d+1}\right) \prod_{i=1}^{k} \exp \left(-c_{4} \frac{h_{i}^{(d+1) / 2}}{k}\right) .
\end{gathered}
$$

Now, we use that

$$
\left(\left|S^{\prime} \cup T^{\prime}\right| !\right)^{w[\xi]}\left(\left|K_{1}\right| !\right)^{w[\xi]} \cdots\left(\left|K_{m}\right| !\right)^{w[\xi]} \leq(k !)^{w[\xi]}
$$


which leads to

$$
\begin{aligned}
& \left|\left(m_{\lambda}\left(\mathbf{x}_{S^{\prime} \cup T^{\prime}}\right)-m_{\lambda}\left(\mathbf{x}_{S^{\prime}}\right) m_{\lambda}\left(\mathbf{x}_{T^{\prime}}\right)\right) m_{\lambda}\left(\mathbf{x}_{K_{1}}\right) \cdots m_{\lambda}\left(\mathbf{x}_{K_{m}}\right)\right| \\
& \quad \leq c_{1} c_{2}^{k} k(k !)^{w[\xi]} \exp \left(-c_{3} d\left(v_{S}, v_{T}\right)^{d+1}\right) \prod_{i=1}^{k} \exp \left(-c_{4} \frac{h_{i}^{(d+1) / 2}}{k}\right) .
\end{aligned}
$$

Together with (17), we have that

$$
\begin{aligned}
& \left|\int_{\sigma(\{S, T\})} \mathrm{d} c_{\lambda}^{k} f^{\otimes k}\right| \\
& \quad \leq c_{1} c_{2}^{k}(k !)^{w[\xi]+1}\|f\|_{\infty}^{k} k \int_{\left(\mathbb{B}^{d}\right)^{k}} \tilde{\mathrm{d}}_{\lambda} \mathbf{x} \exp \left(-c_{3} \delta(\mathbf{x})^{d+1}\right) \prod_{i=1}^{k} \exp \left(-c_{4} \frac{h_{i}^{(d+1) / 2}}{k}\right) .
\end{aligned}
$$

What is left is to bound the integral over $\left(\mathbb{B}^{d}\right)^{k}$ appearing in the last expression. To evaluate it, we can and will assume without loss of generality that the point $x_{1}$ is mapped onto $\left(0, h_{1}\right) \in \mathcal{R}_{\lambda}$ under $\mathscr{T}_{\lambda}$ (this is possible after a suitable rotation of $\eta_{\lambda}$ ). Using this together with the definition of the singular differential, we conclude that

$$
\begin{aligned}
& \int_{\left(\mathbb{B}^{d}\right)^{k}} \tilde{\mathrm{d}}_{\lambda} \operatorname{xexp}\left(-c_{1} \delta(\mathbf{x})^{d+1}\right) \prod_{i=1}^{k} \exp \left(-c_{2} \frac{h_{i}^{(d+1) / 2}}{k}\right) \\
& =\sum_{L_{1}, \ldots, L_{p} \leq[k]} \lambda^{p} \int_{\left(\mathbb{B}^{d}\right)^{p}} \mathrm{~d}\left(x_{1}, \ldots, x_{p}\right) \exp \left(-c_{1} \delta\left(0, v_{2}, \ldots, v_{p}\right)^{d+1}\right) \\
& \quad \times \prod_{i=1}^{p} \exp \left(-c_{2} \frac{\left|L_{i}\right| h_{i}^{(d+1) / 2}}{k}\right) \\
& \leq \sum_{L_{1}, \ldots, L_{p} \leq[k]} \lambda^{p} \int_{\left(\mathbb{B}^{d}\right)^{p}} \mathrm{~d}\left(x_{1}, \ldots, x_{p}\right) \exp \left(-c_{1} \delta\left(0, v_{2}, \ldots, v_{p}\right)^{d+1}\right) \\
& \quad \times \prod_{i=1}^{p} \exp \left(-c_{2} \frac{h_{i}^{(d+1) / 2}}{k}\right) .
\end{aligned}
$$

We now introduce spherical coordinates for $x_{1}$ and use the definition of the scaling transformation $\mathscr{T}_{\lambda}$ for $x_{2}, \ldots, x_{p}$. For the differential elements $\mathrm{d} x_{1}, \ldots, \mathrm{d} x_{p}$ this means that

$$
\mathrm{d} x_{1}=\left(1-\lambda^{-\frac{2}{d+1}} h_{1}\right)^{d-1} \lambda^{-\frac{2}{d+1}} \mathrm{~d} h_{1} \mathcal{H}_{\mathbb{S}^{d-1}}^{d-1}(\mathrm{~d} u)
$$

and that

$$
\mathrm{d} x_{i}=\left(\lambda^{-\frac{1}{d+1}}\right)^{d-1} \lambda^{-\frac{2}{d+1}} \mathrm{~d} h_{i} \mathrm{~d} v_{i}, \quad i \in\{2, \ldots, p\} .
$$


Together with the observation that $p-\frac{2 p}{d+1}-\frac{1}{d+1}(d-1)(p-1)=\frac{d-1}{d+1}$, we see that

$$
\begin{aligned}
\lambda^{p} \int_{\left(\mathbb{B}^{d}\right)^{p}} \mathrm{~d}\left(x_{1}, \ldots, x_{p}\right) \exp \left(-c_{1} \delta\left(0, v_{2}, \ldots, v_{p}\right)^{d+1}\right) \prod_{i=1}^{p} \exp \left(-c_{2} \frac{h_{i}^{(d+1) / 2}}{k}\right) \\
=\lambda^{p} \int_{\mathbb{S}^{d-1}} \mathcal{H}_{\mathbb{S}^{d-1}}^{d-1}(\mathrm{~d} u) \int_{0}^{\lambda^{\frac{2}{d+1}}} \mathrm{~d} h_{1} \ldots \int_{0}^{\lambda^{\frac{2}{d+1}}} \mathrm{~d} h_{p} \int_{\mathscr{T}_{\lambda}\left(\mathbb{S}^{d-1}\right)} \mathrm{d} v_{2} \cdots \int_{\mathscr{T}_{\lambda}\left(\mathbb{S}^{d-1}\right)} \mathrm{d} v_{p} \\
\quad \times \exp \left(-c_{1} \delta\left(0, v_{2}, \ldots, v_{p}\right)^{d+1}\right) \\
\quad \times \prod_{i=1}^{p} \exp \left(-c_{2} \frac{h_{i}^{(d+1) / 2}}{k}\right)\left(1-\lambda^{\left.-\frac{2}{d+1} h_{1}\right)^{d-1} \lambda^{-\frac{1}{d+1}(d-1)(p-1)} \lambda^{-\frac{2 p}{d+1}}}\right. \\
\leq \lambda^{\frac{d-1}{d+1}} \int_{\mathbb{S}^{d-1}} \mathcal{H}_{\mathbb{S}^{d-1}}^{d-1}(\mathrm{~d} u) \int_{0}^{\infty} \mathrm{d} h_{1} \cdots \int_{0}^{\infty} \mathrm{d} h_{p} \int_{\left(\mathbb{R}^{d-1}\right)^{p-1}} \mathrm{~d} \mathbf{v} \\
\quad \times \exp \left(-c_{1} \delta(0, \mathbf{v})^{d+1}\right) \prod_{i=1}^{p} \exp \left(-c_{2} \frac{h_{i}^{(d+1) / 2}}{k}\right) .
\end{aligned}
$$

Since $\int_{\mathbb{S}^{d-1}} \mathcal{H}_{\mathbb{S}^{d-1}}^{d-1}(\mathrm{~d} u)=d \kappa_{d}$, this yields the result.

Fix from now on a partition $\left\{L_{1}, \ldots, L_{p}\right\}$ of $[k]$ into $p \leq k$ blocks. Our next goal is to bound the integral

$$
\int_{\left(\mathbb{R}^{d-1}\right)^{p-1}} \mathrm{~d} \mathbf{v} \exp \left(-c \delta(0, \mathbf{v})^{d+1}\right)
$$

that has shown up in Lemma 5.7, where $c \in(0, \infty)$ is a constant only depending on $d$ and on $\xi$.

Lemma 5.8. We have that

$$
\int_{\left(\mathbb{R}^{d-1}\right)^{p-1}} \mathrm{~d} \mathbf{v} \exp \left(-c \delta(0, \mathbf{v})^{d+1}\right) \leq c_{1} c_{2}^{k} k^{k-2} k ! .
$$

Proof. Using that

$$
\int_{\delta(0, \mathbf{v})^{d+1}}^{\infty} \mathrm{d} t \exp (-c t)=\frac{1}{c} \exp \left(-c \delta(0, \mathbf{v})^{d+1}\right)
$$

together with Fubini's theorem, we obtain

$$
\begin{aligned}
& \int_{\left(\mathbb{R}^{d-1}\right)^{p-1}} \mathrm{~d} \mathbf{v} \exp \left(-c \delta(0, \mathbf{v})^{d+1}\right) \\
& \quad=c \int_{\left(\mathbb{R}^{d-1}\right)^{p-1}} \int_{\delta(0, \mathbf{v})^{d+1}}^{\infty} \mathrm{d} t \mathrm{~d} \mathbf{v} \exp (-c t)
\end{aligned}
$$




$$
\begin{aligned}
& =c \int_{0}^{\infty} \int_{\delta(0, \mathbf{v})^{d+1}<t} \mathrm{~d} \mathbf{v} \mathrm{d} t \exp (-c t) \\
& =c \int_{0}^{\infty} \int_{\delta(0, \mathbf{v})<t} \frac{1}{d+1} \mathrm{~d} \mathbf{v} \mathrm{d} t \exp (-c t) .
\end{aligned}
$$

Now, suppose that $\delta(0, \mathbf{v})<t^{\frac{1}{d+1}}$. Then there is obviously no partition $\{S, T\}$ of $\{0, \mathbf{v}\}$ such that the corresponding separation $d\left(v_{S}, v_{T}\right)$ is bigger than $t^{\frac{1}{d+1}}$. This implies that there exists a tree $\mathcal{T}$ on $\{1, \ldots, p\}$ such that adjacent vertices $v_{i}, v_{j}$ in $\mathcal{T}$ satisfy $\left\|v_{i}-v_{j}\right\|<t^{\frac{1}{d+1}}$. We indicate this property by writing $(0, \mathbf{v}) \prec\left(t^{\frac{1}{d+1}}, \mathcal{T}\right)$ and thus have

$$
\begin{aligned}
\int_{\delta(0, \mathbf{v})<t \frac{1}{d+1}} \mathrm{~d} \mathbf{v} & \leq \sum_{\mathcal{T}} \int_{(0, \mathbf{v}) \prec\left(t \frac{1}{d+1}, \mathcal{T}\right)} \mathrm{d} \mathbf{v} \\
& =\sum_{\mathcal{T}} \operatorname{vol}_{(p-1)(d-1)}\left(\left\{\mathbf{v} \in\left(\mathbb{R}^{d-1}\right)^{p-1}\right\}:(0, \mathbf{v}) \prec\left(t^{\frac{1}{d+1}}, \mathcal{T}\right)\right),
\end{aligned}
$$

where the sum ranges over all trees $\mathcal{T}$ on the edges $\{1, \ldots, p\}$ and the $(p-1)(d-1)$-dimensional volume of the considered set. By the geometry of these trees it follows that

$$
\operatorname{vol}_{(p-1)(d-1)}\left(\left\{\mathbf{v} \in\left(\mathbb{R}^{d-1}\right)^{p-1}\right\}:(0, \mathbf{v}) \prec\left(t^{\frac{1}{d+1}}, \mathcal{T}\right)\right) \leq\left(t^{\frac{d-1}{d+1}} \kappa_{d-1}\right)^{p-1}=t^{\frac{(d-1)(p-1)}{d+1}} \kappa_{d-1}^{p-1} .
$$

Moreover, by Caley's theorem there are exactly $p^{p-2}$ trees on $\{1, \ldots, p\}$. This gives

$$
\int_{\delta(0, \mathbf{v})<t \frac{1}{d+1}} \mathrm{~d} \mathbf{v} \leq p^{p-2} \kappa_{d-1}^{p-1} t^{\frac{(d-1)(p-1)}{d+1}} .
$$

Furthermore, using the Gamma function we see that

$$
\int_{0}^{\infty} \mathrm{d} t t^{\frac{(d-1)(p-1)}{d+1}} \exp (-c t) \leq c_{1} c_{2}^{p} p !
$$

and this leads to

$$
\int_{\left(\mathbb{R}^{d-1}\right)^{p-1}} \mathrm{~d} \mathbf{v} \exp \left(-c \delta(0, \mathbf{v})^{d+1}\right) \leq c_{1} p^{p-2} \kappa_{d-1}^{p-1} \int_{0}^{\infty} \mathrm{d} t t^{\frac{(d-1)(p-1)}{d+1}} \exp \left(-c_{2} t\right) \leq c_{3} c_{4}^{k} k^{k-2} k ! .
$$

This completes the proof.

We get the following final upper bound for the off-diagonal term.

Lemma 5.9. For $\xi \in \Xi$ and $f \in \mathcal{B}\left(\mathbb{B}^{d}\right)$ we have that

$$
\left|\sum_{S, T \leq[k]} \int_{\sigma(\{S, T\})} \mathrm{d} c_{\lambda}^{k} f^{\otimes k}\right| \leq c_{1} c_{2}^{k}\|f\|_{\infty}^{k}(k !)^{w[\xi]+3} k^{k\left(1+\frac{2}{d+1}\right)} k ! \lambda \frac{d-1}{d+1}
$$

for all $\lambda \geq c_{3}$. 
Proof. Combining Lemmas 5.7 and 5.8 with $\int_{0}^{\infty} \mathrm{d} h_{i} \exp \left(-c_{1} \frac{h_{i}^{(d+1) / 2}}{k}\right)=c_{2} k^{\frac{2}{d+1}}$ for all $i \in$ $\{1, \ldots, p\}$, we conclude that

$$
\begin{aligned}
& \left|\sum_{S, T \leq[k]} \int_{\sigma(\{S, T\})} \mathrm{d} c_{\lambda}^{k} f^{\otimes k}\right| \\
& \quad \leq c_{1} c_{2}^{k} k k^{k-2} k ! k^{\frac{2 k}{d+1}}(k !)^{w[\xi]+1}\|f\|_{\infty}^{k} \lambda^{\frac{d-1}{d+1}} \sum_{L_{1}, \ldots, L_{p} \leq[k]} 1 .
\end{aligned}
$$

The number of terms in the sum is bounded by $k !$. Thus,

$$
\begin{aligned}
\left|\sum_{S, T \preceq[k]} \int_{\sigma(\{S, T\})} \mathrm{d} c_{\lambda}^{k} f^{\otimes k}\right| & \leq c_{1} c_{2}^{k}\|f\|_{\infty}^{k}(k !)^{w[\xi]+3} k^{k\left(1+\frac{2}{d+1}\right)-1} \lambda^{\frac{d-1}{d+1}} \\
& \leq c_{1} c_{2}^{k}\|f\|_{\infty}^{k}(k !)^{w[\xi]+3} k^{k\left(1+\frac{2}{d+1}\right)} \lambda^{\frac{d-1}{d+1}}
\end{aligned}
$$

and this completes the argument.

We are now prepared to show our crucial cumulant bound.

Proof of Proposition 5.1. From Lemmas 5.5 and 5.9 we conclude that

$$
\begin{aligned}
\left|\left\langle f^{\otimes k}, c_{\lambda}^{k}\right\rangle\right| & \leq\left|\int_{\Delta} \mathrm{d} c_{\lambda}^{k} f^{\otimes k}\right|+\left|\sum_{S, T \leq[k]} \int_{\sigma(\{S, T\})} \mathrm{d} c_{\lambda}^{k} f^{\otimes k}\right| \\
& \leq c_{1} c_{2}^{k}\|f\|_{\infty}^{k}(k !)^{w[\xi]+3} k^{k\left(1+\frac{2}{d+1}\right)} \lambda^{\frac{d-1}{d+1}}
\end{aligned}
$$

for all sufficiently large $\lambda$. Now, the elementary inequality $\ell^{\ell} \leq \ell ! e^{3 \ell}$, valid for $\ell \in\{3,4, \ldots\}$, implies that

$$
\begin{aligned}
\left|\left\langle f^{\otimes k}, c_{\lambda}^{k}\right\rangle\right| & \leq c_{1} c_{2}^{k}\|f\|_{\infty}^{k}(k !)^{w[\xi]+4+\frac{2}{d+1}} \lambda^{\frac{d-1}{d+1}} \\
& =c_{1} c_{2}^{k}\|f\|_{\infty}^{k}(k !)^{w[\xi]+\beta(d)} \lambda^{\frac{d-1}{d+1}}
\end{aligned}
$$

for all $\lambda \geq c_{3}$, where the definition of the constant $\beta(d)$ given in (1) was also used. The argument is thus complete.

\subsection{Proof of the theorems}

We are now prepared to establish our main results presented in Section 3. For this, we need the following lemma, which is included to make the paper self-contained. By slight abuse of notation, we denote by $c^{k}(X)$ the $k$ th cumulant of a (real-valued) random variable $X$. It is well 
defined if $\mathbb{E}|X|^{k}<\infty$ and is given by

$$
c^{k}(X):=\left.(-\mathfrak{i})^{k} \frac{\mathrm{d}^{k}}{\mathrm{~d} t^{k}} \log \mathbb{E} e^{\mathfrak{i} t X}\right|_{t=0},
$$

where $i$ stands for the imaginary unit.

Lemma 5.10. Let $\left(X_{\lambda}\right)_{\lambda>0}$ be a family of random variables with $\mathbb{E} X_{\lambda}=0$ and $\operatorname{var} X_{\lambda}=1$ for all $\lambda>0$, and suppose that, for all $\lambda \geq \lambda_{0}$,

$$
\left|c^{k}\left(X_{\lambda}\right)\right| \leq(k !)^{1+\gamma} \Delta_{\lambda}^{-(k-2)}
$$

for some $\lambda_{0}>0, \gamma \in[0, \infty), \Delta_{\lambda} \in(0, \infty)$ and all $k \in\{3,4, \ldots\}$.

(i) There exists $b_{1} \in(0, \infty)$ only depending on $\gamma$ such that

$$
\mathbb{P}\left(\left|X_{\lambda}\right| \geq y\right) \leq 2 \exp \left(-\frac{1}{4} \min \left\{\frac{y^{2}}{2^{1+\gamma}},\left(\Delta_{\lambda} y\right)^{\frac{1}{1+\gamma}}\right\}\right)
$$

for all $y \geq 0$ and $\lambda \geq b_{1}$.

(ii) There are constants $b_{2}, b_{3}, b_{4} \in(0, \infty)$ only depending on $\gamma$ such that for $\Delta_{\lambda} \geq b_{2}$ and $0 \leq y \leq b_{3} \Delta_{\lambda}^{1 /(1+2 \gamma)}$,

$$
\begin{aligned}
\left|\log \frac{\mathbb{P}\left(X_{\lambda} \geq y\right)}{1-\Phi(y)}\right| & \leq b_{4}\left(1+y^{3}\right) \Delta_{\lambda}^{-1 /(1+2 \gamma)} \text { and } \\
\left|\log \frac{\mathbb{P}\left(X_{\lambda} \leq-y\right)}{\Phi(-y)}\right| & \leq b_{4}\left(1+y^{3}\right) \Delta_{\lambda}^{-1 /(1+2 \gamma)},
\end{aligned}
$$

where $\Phi(\cdot)$ is the distribution function of a standard Gaussian random variable.

(iii) If $\left(a_{\lambda}\right)_{\lambda>0}$ is such that

$$
\lim _{\lambda \rightarrow \infty} a_{\lambda}=\infty \text { and } \lim _{\lambda \rightarrow \infty} a_{\lambda} \Delta_{\lambda}^{-1 /(1+2 \gamma)},
$$

then $\left(a_{\lambda}^{-1} X_{\lambda}\right)_{\lambda>0}$ satisfies a moderate deviation principle on $\mathbb{R}$ with speed $a_{\lambda}^{2}$ and rate function $I(y)=\frac{y^{2}}{2}$.

Proof. Part (i) is a reformulation of Lemma 2.4 in [37] in a form taken from Lemma 3.9 in [20] with $H=2^{1+\gamma}$ there. The statement in (ii) corresponds to Lemma 2.3 in [37] in a form that we took from Corollary 3.2 in [20]. Finally, the MDP for the family $\left(X_{\lambda}\right)_{\lambda>0}$ is Theorem 1.1 in [19].

We now combine the previous lemma with the cumulant bound established in Proposition 5.1 to give a proof of our main results for Poisson polytopes. 
Proof of Theorems 3.1, 3.2 and 3.5. We let $\xi \in \Xi$ be a key geometric functional and $f \in \mathcal{C}\left(\mathbb{B}^{d}\right)$ with $\left\langle f^{2}, \mathcal{H}_{\mathbb{S}^{d-1}}^{d-1}\right\rangle \neq 0$. Recalling from (10) that in this case $\sigma_{\lambda}^{\xi}[f] \geq c_{1}\left\langle f^{2}, \mathcal{H}_{\mathbb{S}^{d-1}}^{d-1}\right\rangle^{\frac{1}{2}} \lambda^{\frac{d-1}{2(d+1)}}$, we see in view of Proposition 5.1 that, for $k \in\{3,4, \ldots\}$,

$$
\begin{aligned}
\frac{\left|\left\langle f^{\otimes k}, c_{\lambda}^{k}\right\rangle\right|}{\left(\sigma_{\lambda}^{\xi}[f]\right)^{k}} & \leq c_{1} c_{2}^{k}\|f\|_{\infty}^{k} \lambda^{\frac{d-1}{d+1}}(k !)^{w[\xi]+\beta(d)}\left(c_{3}\left\langle f^{2}, \mathcal{H}_{\mathbb{S}^{d-1}}^{d-1}\right\rangle^{\frac{1}{2}} \lambda^{\frac{d-1}{2(d+1)}}\right)^{-k} \\
& \leq(k !)^{w[\xi]+\beta(d)} c_{4} \lambda^{\frac{d-1}{d+1}}\left(\frac{c_{5}\|f\|_{\infty}}{c_{6}\left\langle f^{2}, \mathcal{H}_{\mathbb{S}^{d-1}}^{d-1}\right\rangle^{\frac{1}{2}} \lambda^{\frac{d-1}{2(d+1)}}}\right)^{k} \\
& =(k !)^{w[\xi]+\beta(d)}\left(c_{9} \lambda^{\frac{d-1}{2(d+1)}}\right)^{-(k-2)}
\end{aligned}
$$

where $c_{9}:=c_{7}\left(c_{5}\|f\|_{\infty} \max \left\{1, c_{8}\right\}\right)^{-1}$ with $c_{7}:=c_{6}\left\langle f^{2}, \mathcal{H}_{\mathbb{S}^{d-1}}^{d-1}\right\rangle^{\frac{1}{2}}$ and $c_{8}:=c_{1} c_{2}^{2} c_{7}^{-2}\|f\|_{\infty}^{2}$ is a constant depending only on $d, \xi$ and $f$. Now, put

$$
\gamma:=w[\xi]+\beta(d)-1 \quad \text { and } \quad \Delta_{\lambda}:=c_{9} \lambda^{\frac{d-1}{2(d+1)}}
$$

and apply Lemma 5.10 to the random variables $X_{\lambda}:=\left(\sigma_{\lambda}^{\xi}[f]\right)^{-1}\left\langle f, \bar{\mu}_{\lambda}^{\xi}\right\rangle$. The results then follow and the proof is complete.

Proof of Theorem 3.6. Theorem 3.6 follows by the same argument as Theorem 1.5 in [20] follows from Theorem 1.4 ibidem with the class $\mathcal{B}\left(\mathbb{B}^{d}\right)$ replaced by $\mathcal{C}\left(\mathbb{S}^{d-1}\right)$. For this reason, details are omitted.

\section{Acknowledgements}

We would like to thank Matthias Reitzner (University of Osnabrück) for helpful discussions and for useful hints and comments to an earlier version of this paper. In addition, our thanks go to an anonymous referee for her/his highly valuable suggestions.

\section{References}

[1] Affentranger, F. (1988). The expected volume of a random polytope in a ball. J. Microscopy 151 277-287.

[2] Bárány, I. (1989). Intrinsic volumes and $f$-vectors of random polytopes. Math. Ann. 285 671-699. MR1027765

[3] Bárány, I. (1992). Random polytopes in smooth convex bodies. Mathematika 39 81-92. MR1176473

[4] Bárány, I. (2007). Random polytopes, convex bodies, and approximation. In Stochastic Geometry (W. Weil, ed.). Lecture Notes in Math. 1892 77-118. Berlin: Springer. MR2327291

[5] Bárány, I. and Reitzner, M. (2010). On the variance of random polytopes. Adv. Math. 225 1986-2001. MR2680197

[6] Bárány, I. and Reitzner, M. (2010). Poisson polytopes. Ann. Probab. 38 1507-1531. MR2663635 
[7] Baryshnikov, Yu. and Yukich, J.E. (2005). Gaussian limits for random measures in geometric probability. Ann. Appl. Probab. 15 213-253. MR2115042

[8] Borgwardt, K.-H. (1987). The Simplex Method: A Probabilistic Analysis. Algorithms and Combinatorics: Study and Research Texts 1. Berlin: Springer. MR0868467

[9] Böröczky, K.J. Jr., Hoffmann, L.M. and Hug, D. (2008). Expectation of intrinsic volumes of random polytopes. Period. Math. Hungar. 57 143-164. MR2469601

[10] Brazitikos, S., Giannopoulos, A., Valettas, P. and Vritsiou, B.-H. (2014). Geometry of Isotropic Convex Bodies. Mathematical Surveys and Monographs 196. Providence, RI: Amer. Math. Soc. MR3185453

[11] Buchta, C. and Müller, J. (1984). Random polytopes in a ball. J. Appl. Probab. 21 753-762. MR0766813

[12] Cabo, A.J. and Groeneboom, P. (1994). Limit theorems for functionals of convex hulls. Probab. Theory Related Fields 100 31-55. MR1292189

[13] Calka, P. (2013). Asymptotic methods for random tessellations. In Stochastic Geometry, Spatial Statistics and Random Fields (E. Spodarev, ed.). Lecture Notes in Math. 2068 183-204. Heidelberg: Springer. MR3059648

[14] Calka, P. and Schreiber, T. (2005). Limit theorems for the typical Poisson-Voronoi cell and the Crofton cell with a large inradius. Ann. Probab. 33 1625-1642. MR2150201

[15] Calka, P. and Schreiber, T. (2006). Large deviation probabilities for the number of vertices of random polytopes in the ball. Adv. in Appl. Probab. 38 47-58. MR2213963

[16] Calka, P., Schreiber, T. and Yukich, J.E. (2013). Brownian limits, local limits and variance asymptotics for convex hulls in the ball. Ann. Probab. 41 50-108. MR3059193

[17] Calka, P. and Yukich, J.E. (2014). Variance asymptotics for random polytopes in smooth convex bodies. Probab. Theory Related Fields 158 435-463. MR3152787

[18] Dembo, A. and Zeitouni, O. (2010). Large Deviations Techniques and Applications. Stochastic Modelling and Applied Probability 38. Berlin: Springer. MR2571413

[19] Döring, H. and Eichelsbacher, P. (2013). Moderate deviations via cumulants. J. Theoret. Probab. 26 360-385. MR3055808

[20] Eichelsbacher, P., Raič, M. and Schreiber, T. (2015). Moderate deviations for stabilizing functionals in geometric probability. Ann. Inst. Henri Poincaré Probab. Stat. 51 89-128. MR3300965

[21] Groeneboom, P. (1988). Limit theorems for convex hulls. Probab. Theory Related Fields 79 327-368. MR0959514

[22] Hörrmann, J. and Hug, D. (2014). On the volume of the zero cell of a class of isotropic Poisson hyperplane tessellations. Adv. in Appl. Probab. 46 622-642. MR3254334

[23] Hörrmann, J., Hug, D., Reitzner, M. and Thäle, C. (2015). Poisson polyhedra in high dimensions. Adv. Math. 281 1-39. MR3366836

[24] Hsing, T. (1994). On the asymptotic distribution of the area outside a random convex hull in a disk. Ann. Appl. Probab. 4 478-493. MR1272736

[25] Hueter, I. (1999). Limit theorems for the convex hull of random points in higher dimensions. Trans. Amer. Math. Soc. 351 4337-4363. MR1670156

[26] Hug, D. (2013). Random polytopes. In Stochastic Geometry, Spatial Statistics and Random Fields (E. Spodarev, ed.). Lecture Notes in Math. 2068 205-238. Heidelberg: Springer. MR3059649

[27] Hug, D. and Schneider, R. (2007). Asymptotic shapes of large cells in random tessellations. Geom. Funct. Anal. 17 156-191. MR2306655

[28] Küfer, K.-H. (1994). On the approximation of a ball by random polytopes. Adv. in Appl. Probab. 26 876-892. MR1303867

[29] Müller, J.S. (1990). Approximation of a ball by random polytopes. J. Approx. Theory 63 198-209. MR1079850 
[30] Pardon, J. (2011). Central limit theorems for random polygons in an arbitrary convex set. Ann. Probab. 39 881-903. MR2789578

[31] Reitzner, M. (2003). Random polytopes and the Efron-Stein jackknife inequality. Ann. Probab. 31 2136-2166. MR2016615

[32] Reitzner, M. (2004). Stochastic approximation of smooth convex bodies. Mathematika 51 11-29. MR2220208

[33] Reitzner, M. (2005). The combinatorial structure of random polytopes. Adv. Math. 191 178-208. MR2102847

[34] Reitzner, M. (2005). Central limit theorems for random polytopes. Probab. Theory Related Fields 133 483-507. MR2197111

[35] Reitzner, M. (2010). Random polytopes. In New Perspectives in Stochastic Geometry (W.S. Kendall and I. Molchanov, eds.) 45-76. Oxford: Oxford Univ. Press. MR2654675

[36] Rényi, A. and Sulanke, R. (1963). Über die konvexe Hülle von $n$ zufällig gewählten Punkten. Z. Wahrsch. Verw. Gebiete 2 75-84. MR0156262

[37] Saulis, L. and Statulevičius, V.A. (1991). Limit Theorems for Large Deviations. Mathematics and Its Applications (Soviet Series) 73. Dordrecht: Kluwer Academic. MR1171883

[38] Schneider, R. and Weil, W. (2008). Stochastic and Integral Geometry. Probability and Its Applications (New York). Berlin: Springer. MR2455326

[39] Schreiber, T. (2002). Variance asymptotics and central limit theorems for volumes of unions of random closed sets. Adv. in Appl. Probab. 34 520-539. MR1929596

[40] Schreiber, T. (2003). Asymptotic geometry of high-density smooth-grained Boolean models in bounded domains. Adv. in Appl. Probab. 35 913-936. MR2014262

[41] Schreiber, T. and Yukich, J.E. (2008). Variance asymptotics and central limit theorems for generalized growth processes with applications to convex hulls and maximal points. Ann. Probab. 36 363-396. MR2370608

[42] Schütt, C. (1994). Random polytopes and affine surface area. Math. Nachr. 170 227-249. MR1302377

[43] Vu, V. (2006). Central limit theorems for random polytopes in a smooth convex set. Adv. Math. 207 221-243. MR2264072

[44] Vu, V.H. (2005). Sharp concentration of random polytopes. Geom. Funct. Anal. 15 1284-1318. MR2221249

Received May 2016 and revised January 2017 